\title{
Dynamics of a Family of Piecewise-Linear Area-Preserving Plane Maps II. Invariant Circles
}

\author{
Jeffrey C. Lagarias \\ Department of Mathematics \\ University of Michigan \\ Ann Arbor, MI 48109-1043 \\ email: lagarias@umich.edu \\ Eric Rains \\ Department of Mathematics \\ University of California-Davis \\ Davis, CA 95616-8633 \\ email: rains@math.ucdavis.edu
}

(July 1, 2005 version)

\begin{abstract}
This paper studies the behavior under iteration of the maps $T_{a b}(x, y)=\left(F_{a b}(x)-y, x\right)$ of the plane $\mathbb{R}^{2}$, in which $F_{a b}(x)=a x$ if $x \geq 0$ and $b x$ if $x<0$. The orbits under iteration correspond to solutions of the difference equation $x_{n+2}=1 / 2(a-b)\left|x_{n+1}\right|+1 / 2(a+b) x_{n+1}-x_{n}$. This family of piecewise-linear maps of the plane has the parameter space $(a, b) \in \mathbb{R}^{2}$. These maps are area-preserving homeomorphisms of $\mathbb{R}^{2}$ that map rays from the origin into rays from the origin. We show the existence of special parameter values where $T_{a b}$ has every nonzero orbit contained in an invariant circle with an irrational rotation number, with invariant circles that are piecewise unions of arcs of conic sections. Numerical experiments indicate the possible existence of invariant circles for many other parameter values.
\end{abstract}

Keywords: area preserving map, iterated map, symbolic dynamics AMS Subject Classification: Primary: 37E30 Secondary: 52C23, 82D30

\section{Introduction}

We continue the study the behavior under iteration of the two parameter family of piecewiselinear homeomorphisms of $\mathbb{R}^{2}$ given by

$$
T_{a b}(x, y)= \begin{cases}(a x-y, x) & \text { if } \quad x \geq 0 \\ (b x-y, x) & \text { if } \quad x<0\end{cases}
$$


The parameter space is $(a, b) \in \mathbb{R}^{2}$. This map can be written

$$
T_{a b}(x, y)=\left[\begin{array}{cc}
F_{a b}(x) & -1 \\
1 & 0
\end{array}\right]\left[\begin{array}{l}
x \\
y
\end{array}\right]
$$

in which

$$
F_{a b}(x)=\left\{\begin{array}{lll}
a & \text { if } & x \geq 0 \\
b & \text { if } & x<0
\end{array}\right.
$$

we view elements of $(x, y) \in \mathbb{R}^{2}$ as column vectors. The formula (1.2) shows that $T_{a b}(x, y)$ is a homeomorphism, since

$$
\begin{aligned}
T_{a b}^{-1}(x, y) & =\left[\begin{array}{cc}
F_{a b}(y) & -1 \\
1 & 0
\end{array}\right]^{-1}\left[\begin{array}{l}
x \\
y
\end{array}\right] \\
& =\left[\begin{array}{cc}
0 & 1 \\
-1 & F_{a b}(y)
\end{array}\right]\left[\begin{array}{l}
x \\
y
\end{array}\right] .
\end{aligned}
$$

It preserves the area form $d \omega=d x \wedge d y$, and it also maps rays from the origin into rays from the origin. The orbits of the iteration are $T_{a b}\left(x_{n+1}, x_{n}\right)=\left(x_{n+2}, x_{n+1}\right)$, in which the quantities $x_{n}$ satisfy the difference equation

$$
x_{n+2}=\mu\left|x_{n+1}\right|+\nu x_{n+1}-x_{n} .
$$

in which $\mu=1 / 2(a-b)$ and $\nu=1 / 2(a+b)$. For certain purposes the $(\mu, \nu)$ coordinate system is more convenient than the $(a, b)$ coordinate system (see part III), but in this paper we shall exclusively use the $(a, b)$ coordinate system.

The main result of this paper is the characterization of certain one-parameter families of $T_{a b}$ whose generic members exhibit quasiperiodic motion under iteration, so have bounded orbits (Theorem 2.2). These families are those for which $(0,1)$ and $(0,-1)$ fall in the same orbit with a fixed symbolic dynamics between their occurrences, and which contain a parameter value having an irrational rotation number. We show that generic members of any such family have each orbit contained in an invariant circle which is a finite union of arcs of conic sections. We rigorously establish for specific examples that the irrational rotation number property holds (Examples 4.1 and 4.2).

Recall that $\Omega_{S B}$ is the set of all parameter values for which the map $T_{a b}$ has at least one nonzero bounded orbit, $\Omega_{B}$ is the the set of paramenter values having all orbits bounded and $\Omega_{R}$ is the set of parameter values for which $T_{a b}$ is topologically conjugate to a rotation of the plane, or equivalently, having an invariant circle. (We regard a purely periodic map as having an invariant circle, which may be constructed artificially from a suitable union of orbits, as all orbits are finite.) Our results here have a bearing on $\Omega_{S B}$, because there appears to be a close relation between the narrow set of parameter values having piecewise conic invariant circles, which we show has two-dimensional Lebesgue measure zero, and the larger set $\Omega_{S B}$. Let $\Omega_{P}$ denote the set of parameter values $(a, b)$ for which $T_{a b}$ is periodic, and let $\Omega_{Q}(\mathrm{Q}=$ "quadratic") denote the set of parameter values having a piecewise conic invariant circle as prescribed by Theorem 3.4. Then we have the inclusions

$$
\Omega_{P} \subset \Omega P \cup \Omega_{Q} \subseteq \Omega_{R}=\Omega_{B} \subseteq \Omega_{S B} .
$$


We formulate the following conjecture.

Conjecture A. The set $\Omega_{P} \cup \Omega_{Q}$ of pairs $(a, b)$ for which $T_{a b}$ is either periodic or has a piecewise conic invariant circle is dense in $\Omega_{S B}$.

A closely related conjecture was made by Bedford, Bullett and Rippon [3, to the effect that the parameter set of purely periodic maps $\Omega_{P}$ is dense in $\Omega_{B}$. In part III we will show that $\Omega_{S B}$ is a closed set, and it is natural to expect that it is the closure of $\Omega_{B}$, though we have not established this. Combining this expectation with the conjecture of [3], would result in the conjecture that $\Omega_{P}$ is dense in $\Omega_{S B}$.

In $\S 5$ we show that $\Omega_{P} \cup \Omega_{Q}$ has Hausdorff dimension 1 , hence has two-dimensional Lebesgue measure zero. We also present nonrigorous numerical evidence in $\S 4$ of invariant circles for a variety of parameter values which appear to fall outside the set $\Omega_{P} \cup \Omega_{Q}$.

There has been previous work concerning invariant circles for these maps. In $1986 \mathrm{M}$. Herman [6, Theorem VIII.5.1] proved results implying that any map $T_{a b}$ having an irrational rotation number $r\left(S_{a b}\right)=r$ such that $r$ has bounded partial quotients in its continued fraction expansion is topologically conjugate to a rotation of the plane, so has an invariant circle. Herman's results also supply some justification for the numerical observations that are noted in $\S 4$; his set of allowed parameter values presumably has Hausdorff dimension 2, but with twodimensional Lebesgue measure zero. It undoubtably include parameter values outside $\Omega_{P} \cup \Omega_{Q}$.

In 1995 Beardon, Bullett and Rippon [3, p.673] announced without proof as work to come results related to those appearing here. They asserted that maps $T_{a b}$ mapping $(0,1)$ to $(0,-1)$ after a finite number of steps with a fixed symbolic itinerary should give an algebraic curve of parameter values on which there is an open subset of values where the map is topologically conjugate to a rotation of the plane, compare our Theorem 2.2. Example 4.3 below shows that their assertion requires additional hypotheses (such as nonconstancy of the rotation number along the curve) for the stated conclusion to hold. It seems clear they had knowledge of various ideas presented here; however they made no comment regarding the nature of the invariant circles.

Notation. We write $\mathbf{v}=\left(\mathbf{v}_{x}, \mathbf{v}_{y}\right) \in \mathbb{R}^{2}$, to be viewed as a column vector. An interval $\left[\mathbf{v}_{1}, \mathbf{v}_{2}\right)$ of the unit circle, or corresponding sector $\mathbb{R}^{+}\left[\mathbf{v}_{1}, \mathbf{v}_{2}\right)$ of the plane $\mathbb{R}^{2}$, is the one specified by going counterclockwise from $\mathbf{v}_{1}$ to $\mathbf{v}_{2}$.

Acknowledgments. We did most of the work reported in this paper while employed at AT\&T Bell Labs; most results of this paper were obtained during the summer of 1993. We thank M. Kontsevich for bringing the work of Beardon, Bullett and Rippon [3] to our attention.

\section{Summary of Results}

It is convenient to represent the action of $T_{a b}$, acting on row vectors $\mathbf{v}_{n}=\left(x_{n+1}, x_{n}\right)$ as

$$
T_{n}\left(\mathbf{v}_{0}\right)=\left(x_{n+1}, x_{n}\right)=M_{n}\left(\mathbf{v}_{0}\right)\left(x_{1}, x_{0}\right)
$$


in which

$$
M_{n}\left(\mathbf{v}_{0}\right)=\prod_{i=1}^{n}\left[\begin{array}{cc}
F_{a b}\left(x_{i}\right) & -1 \\
1 & 0
\end{array}\right]:=\left[\begin{array}{cc}
F_{a b}\left(x_{n}\right) & -1 \\
1 & 0
\end{array}\right] \ldots\left[\begin{array}{cc}
F_{a b}\left(x_{2}\right) & -1 \\
1 & 0
\end{array}\right]\left[\begin{array}{cc}
F_{a b}\left(x_{1}\right) & -1 \\
1 & 0
\end{array}\right] .
$$

Conjugation by the involution $J_{0}:(x, y) \rightarrow(-x,-y)$ gives

$$
T_{b a}=J_{0}^{-1} \circ T_{a b} \circ J_{0}
$$

Thus, in studying dynamics, without loss of generality we can restrict to the closed half-space $\{(a, b): a \geq b\}$ of the $(a, b)$ parameter space.

The associated rotation map $S_{a b}: S^{1} \rightarrow S^{1}$ is given by

$$
S_{a b}\left(e^{i \theta}\right):=\frac{T_{a b}\left(e^{i \theta}\right)}{\left|T_{a b}\left(e^{i \theta}\right)\right|}
$$

It has a well-defined rotation number $r\left(S_{a b}\right)$, which is counterclockwise rotation, and was shown in part I to always lie in the closed interval $[0,1 / 2]$.

In part I we classified the dynamics of $T_{a b}$ in the case where the rotation number $r\left(S_{a b}\right)$ is rational, as follows [7, Theorem 2.4].

Theorem 2.1 If the rotation number $r\left(S_{a b}\right)$ is rational, then $S_{a b}$ has a periodic orbit, and one of the following three possibilities occurs.

(i) $S_{a b}$ has exactly one periodic orbit. Then $T_{a b}$ has exactly one periodic orbit (up to scaling) and all other orbits diverge in modulus to $+\infty$ as $n \rightarrow \pm \infty$.

(ii) $S_{a b}$ has exactly two periodic orbits. Then $T_{a b}$ has no periodic orbits. All orbits of $T_{a b}$ diverge in modulus to $+\infty$ as $n \rightarrow \pm \infty$, with the exception of orbits lying over the two periodic orbits of $S_{a b}$. These exceptional orbits have modulus diverging to $+\infty$ in one direction and to 0 in the other direction, with forward divergence for one, and backward divergence for the other.

(iii) $S_{a b}$ has at least three periodic orbits. Then $T_{a b}$ is of finite order, i.e. $T_{a b}^{(k)}=I$ for some $k \geq 1$, and all its orbits are periodic.

In this paper we are mainly concerned with parameter values where the rotation number is irrational, but we will need the result above. The main result of this paper is that there exist parameter values $(a, b)$ with irrational rotation number for which $T_{a b}$ has invariant circles with a striking structure.

Theorem 2.2 Suppose that the rotation number $r\left(S_{a b}\right)$ is irrational, and that the $S_{a b}$ orbit of $(0,1)$ contains $(0,-1)$. Then the following hold.

(1) The $T_{a b}$ orbit of $(0,1)$ contains $(0,-1)$.

(2) The closure of every (nonzero) orbit of $T_{a b}$ is an invariant circle, which is a piecewise union of arcs of conic sections. The conic sections occurring in such an invariant circle are all of the same type, either ellipses, hyperbolas or straight lines.

This result follows from Theorem 3.4 which gives more detailed information about the the number of conic pieces in such an invariant circle. 
In $\S 4$ we give examples exhibiting invariant circles of all the types allowed by Theorem 2.2 An example is $a=2^{1 / 4}, b=-2^{1 / 4}$, which has irrational rotation number and whose invariant circles are the union of eight segments of ellipses. We actually exhibit one-parameter families whose generic members have the required properties. Here "generic" means irrational rotation number; these one-parameter families include values $T_{a b}$ with rational rotation number, where $T_{a b}$ is a periodic map. In the Appendix we verify the conditions of Theorem 3.4 hold in these examples, and exhibit some specific parameter values where the rotation number is irrational. In some cases we are able to show the rotation number is transcendental, using Baker's method in transcendental number theory. In $\S 4$ we also give a variety of computer plots for parameter values not covered by Theorem 2.2 which numerically appear to give invariant circles. We have no rigorous proof of the existence of non piecewise-conic invariant circles, however.

In $\S 5$ we show that the set $\Omega_{Q}$ has Hausdorff dimension 1 and discuss supporting evidence for Conjecture A. We conclude with a discussion of the possiblity of $T_{a b}$ with associated circle map having irrational rotation number, but with $T_{a b}$ not being conjugate to a rotation of the plane.

\section{Irrational Rotation Number}

In this paper we study maps in the irrational rotation case by considering their first-return maps to suitable sectors of the plane.

Theorem 3.1 Suppose that the rotation number $r\left(S_{a b}\right)$ is irrational.

(1) For any half-open sector $J=\mathbb{R}^{+}\left[\mathbf{v}, \mathbf{v}^{\prime}\right)$ the first return map $T_{J}^{(1)}: J \rightarrow J$ of $T_{a b}$ to $J$ is piecewise linear with at most five pieces.

(2) Let $m_{+}, m_{-}, n_{+}, n_{-}$be nonnegative integers, and set $\mathcal{O}:=\mathcal{O}_{1} \cup \mathcal{O}_{2} \cup \mathcal{O}_{3} \cup \mathcal{O}_{4}$, where:

$$
\begin{aligned}
\mathcal{O}_{1} & =\left\{T_{a b}^{(1+i)}(0,1): 0 \leq i<m_{+}\right\} \\
\mathcal{O}_{2} & =\left\{T_{a b}^{(-i)}(0,1): 0 \leq i<m_{-}\right\} \\
\mathcal{O}_{3} & =\left\{T_{a b}^{(1+i)}(0,-1): 0 \leq i<n_{+}\right\} \\
\mathcal{O}_{4} & =\left\{T_{a b}^{(-i)}(0,-1): 0 \leq i<n_{-}\right\} .
\end{aligned}
$$

Let $J=\mathbb{R}^{+}\left[\mathbf{v}, \mathbf{v}^{\prime}\right)$ be any half-open sector determined by two elements $\mathbf{v}, \mathbf{v}^{\prime} \in \mathcal{O}$ such that $J$ contains no points of $\mathcal{O}$ in its interior. Then the first return map $T_{J}: J \rightarrow J$ is piecewise linear with at most three pieces.

Proof. (1) Note first that for any half-open sector $J$, the first return map for $T_{a b}$ is welldefined, since the assumption that $S_{a b}$ has irrational rotation number implies that the orbit of any point under $S_{a b}$ is dense.

Let $\mathbf{v}_{1}$ be the first preimage $T_{a b}^{(-i)}(0,1), i \geq 0$, of $(0,1)$ that falls in $J$; let $\mathbf{v}_{2}$ be the first preimage of $(0,-1)$ with the same property. Let $\mathbf{v}_{3}$ be the first point of the form $T_{a b}^{(-i)}(\mathbf{v})$, $i>0$ that falls in $J$, and let $\mathbf{v}_{4}$ be the first point of the form $T_{a b}^{(-i)}\left(\mathbf{v}^{\prime}\right), i \geq 0$ that falls in $J$. The $\mathbf{v}_{i}$ split $J$ into at most five intervals. We claim that the first return map for $T_{a b}$ on the corresponding sector is linear on each corresponding subsector. 
Consider first the (at most) three half-open subsectors into which $J$ is subdivided by $\mathbf{v}_{3}$ and $\mathbf{v}_{4}$. The number of steps until the first return will be constant on each subsector, since between any two points that take a different number of steps to return, there must be a point that hits the boundary of $J$ at or before the first return. If $\mathbf{v}_{5}$ were such a point other than $\mathbf{v}_{3}$ or $\mathbf{v}_{4}$, then the iterates of $\mathbf{v}_{5}$ would hit the ray determined by $\mathbf{v}_{3}$ or $\mathbf{v}_{4}$ before hitting the ray determined by $\mathbf{v}$ or $\mathbf{v}^{\prime}$, which gives a contradiction, since hitting $\mathbf{v}_{3}$ or $\mathbf{v}_{4}$ constitutes a return to $J$.

Now, we restrict our attention to one of these three subsectors, on which the number of iterations until return to $J$ is constant. For any two points in the subsector whose iterates until first return to $J$ are always on the same side of the $y$-axis, the first return map on $J$ is linear on the sector they induce. Thus the only points where linearity can fail are points which hit the $y$-axis on iteration before their first return, namely $\mathbf{v}_{1}$ and $\mathbf{v}_{2}$. (Any other such point on $J$ would hit the ray determined by $\mathbf{v}_{1}$ or $\mathbf{v}_{2}$ before it hit the $y$-axis, a contradiction.) Thus, the subsector between each two neighboring such $\mathbf{v}_{i}$ is a subsector on which the first return map on $J$ is linear, and (1) follows.

(2) First note that $T_{a b}(0,1)=(-1,0)$; similarly $T_{a b}(0,-1)=(1,0)$. Suppose now that $\mathbf{v} \in \mathcal{O}_{1}$. by definition $\mathbf{v}_{3}$ is the first strict preimage of $\mathbf{v}$ that falls in $J$. This preimage cannot belong to $\mathcal{O}_{1}$ because by hypothesis the only point of $\mathcal{O}$ in the half-open interval $J$ is the endpoint $\mathbf{v}$. Thus this preimage is a strict preimage of $(-1,0)$, hence is $(0,1)$ or a preimage, so we conclude that $\mathbf{v}_{3}=\mathbf{v}_{1}$.

We deduce the following facts by similar reasoning: (1) If $\mathbf{v} \in \mathcal{O}_{2}$ then $\mathbf{v}_{1}=\mathbf{v}$; (2) If $\mathbf{v} \in \mathcal{O}_{3}$ then $\mathbf{v}_{3}=\mathbf{v}_{2} ;(3)$ If $\mathbf{v} \in \mathcal{O}_{4}$ then $\mathbf{v}_{2}=\mathbf{v}$.

The preimage $\mathbf{v}_{4}$ of $\mathbf{v}^{\prime}$ must also be equal to one of these other values. We distinguish three cases: (a) $\mathbf{v}$ and $\mathbf{v}^{\prime}$ are in the same orbit, with $\mathbf{v}$ coming first, (b) $\mathbf{v}$ and $\mathbf{v}^{\prime}$ are in the same orbit, with $\mathbf{v}^{\prime}$ coming first, (c) $\mathbf{v}$ and $\mathbf{v}^{\prime}$ are in different orbits. In case (a), $\mathbf{v}_{4}=\mathbf{v}$, while in case (b), $\mathbf{v}_{4}=\mathbf{v}_{3}$. In case (c) one deduces that if $\mathbf{v}^{\prime} \in \mathcal{O}_{1} \cup \mathcal{O}_{2}$ then $\mathbf{v}_{4}=\mathbf{v}_{1}$, while if $\mathbf{v}^{\prime} \in \mathcal{O}_{3} \cup \mathcal{O}_{4}$ then $\mathbf{v}_{4}=\mathbf{v}_{2}$. In all cases the five points $\mathbf{v}, \mathbf{v}_{1}, \mathbf{v}_{2}, \mathbf{v}_{3}, \mathbf{v}_{4}$ take at most three different values, and therefore $T_{J}$ has at most three linear pieces on $\mathbb{R}^{+}[J]$.

We next consider the special case where the $T_{a b}$-orbit of $(0,1)$ contains $(0,-1)$. To analyze this case we recall the following result from part I [7, Theorem 3.4].

Theorem 3.2 Let $n \geq 1$ and suppose that $S_{a b}^{(n)}(0,1)=(0, \pm 1)$, or $S_{a b}^{(n)}(0,-1)=(0, \pm 1)$, Then one of the following two relations holds:

$$
\begin{gathered}
T_{a b}^{(n)}(0,1)=(0, \lambda), \\
T_{a b}^{(n)}(0,-1)=\left(0,-\lambda^{-1}\right) .
\end{gathered}
$$

where $\lambda$ is a nonzero real number.

(i) If $\lambda>0$, then both relations above hold. In addition,

$$
T_{a b}^{(n)}(-1,0)=(-\lambda, 0) \quad \text { and } \quad T_{a b}^{(n)}(1,0)=\left(\lambda^{-1}, 0\right) .
$$

The rotation number $r\left(S_{a b}\right)$ is rational. 
(ii) If $\lambda<0$, then necessarily $\lambda=-1$. In the first case

$$
T_{a b}^{(n)}(0,1)=(0,-1) \quad \text { and } \quad T_{a b}^{(n)}(-1,0)=(1,0),
$$

while in the second case,

$$
T_{a b}^{(n)}(0,-1)=(0,1) \quad \text { and } \quad T_{a b}^{(n)}(1,0)=(-1,0) .
$$

The rotation number $r\left(S_{a b}\right)$ can be irrational or rational.

We now show that the $T_{a b}$-orbit of $(0,1)$ contains $(0,-1)$, then one can obtain sectors on which the first return map is piecewise linear with at most two pieces, and a surprising thing happens:

Theorem 3.3 Suppose that $S_{a b}$ has irrational rotation number, and that $(0,1)$ and $(0,-1)$ are in the same $T_{a b}$-orbit. Let $\mathcal{O}=\left\{T_{a b}^{(j)}\left(\mathbf{v}_{0}\right): 1 \leq j \leq m\right\}$ be any finite segment of that orbit which includes all of the points strictly between $(0,1)$ and $(0,-1)$, as well as whichever of $(0,1)$ and $(0,-1)$ comes last in the orbit. If $J=\mathbb{R}^{+}\left[\mathbf{v}, \mathbf{v}^{\prime}\right)$ is a half-open sector determined by two members of $\mathcal{O}$ containing no elements of $\mathcal{O}$ in its interior, then the first return map $T_{J}$ on $J$ is piecewise linear with exactly two pieces, and the linear transformations corresponding to the two pieces commute.

Proof. Let $J=\mathbb{R}^{+}\left[\mathbf{v}, \mathbf{v}^{\prime}\right)$ be a sector determined by two members of $\mathcal{O}$, such that no element of $\mathcal{O}$ lies in its interior. We show that the first return map $T_{J}$ of $T_{a b}$ on the sector $J$ is piecewise linear with exactly two pieces. Let $T_{a b}^{(n)}(0,1)=(0,-1)$. We first treat the case $n>0$, when $(0,1)$ comes earlier in the orbit than $(0,-1)$. In the notation of Theorem 3.1 we take $\mathcal{O}_{i}$ as large as possible inside $\mathcal{O}$, and obtain the following relations: $\mathcal{O}_{2} \subset \mathcal{O}_{4}, \mathcal{O}_{3} \subset \mathcal{O}_{1}$, and $\mathcal{O}_{1} \cup \mathcal{O}_{2}=\mathcal{O}_{3} \cup \mathcal{O}_{4}$. Theorem 3.1 (ii) states that the first return map $T_{J}$ is piecewise linear with at most three pieces. We show that the relations between the sets $\mathcal{O}_{i}$ cause two of the pieces to become identified. Indeed, from the proof of Theorem 3.1(ii), we have the following three possibilities:

(a) $\mathbf{v} \in \mathcal{O}_{1} \cap \mathcal{O}_{4}: \mathbf{v}_{3}=\mathbf{v}_{1}, \mathbf{v}_{2}=\mathbf{v}$.

(b) $\mathbf{v} \in \mathcal{O}_{2} \cap \mathcal{O}_{4}: \mathbf{v}_{2}=\mathbf{v}_{1}=\mathbf{v}$.

(c) $\mathbf{v} \in \mathcal{O}_{3} \cap \mathcal{O}_{1}: \mathbf{v}_{3}=\mathbf{v}_{2}=\mathbf{v}_{1}$.

In case (a), $\mathbf{v}_{4}=\mathbf{v}$, while in case (b), $\mathbf{v}_{4}=\mathbf{v}_{3}$; case (c) is excluded, since $\mathbf{v}$ and $\mathbf{v}^{\prime}$ are in the same orbit. Thus the five points $\mathbf{v}, \mathbf{v}_{1}, \mathbf{v}_{2}, \mathbf{v}_{3}, \mathbf{v}_{4}$ take on only two values ( $\mathbf{v}$ and $\mathbf{v}_{3}$ ), which divides the sector $J$ into two pieces. We cannot have a further collapse to one piece because the equality $\mathbf{v}=\mathbf{v}_{3}$ would make $\mathbf{v}$ a periodic point of $S_{\mu \nu}$, whence $S_{\mu \nu}$ has a rational rotation number, contradicting the irrational rotation hypothesis. We conclude that $T_{J}$ is piecewise linear on the sector $J$ with exactly two pieces.

In the other case $n<0$ we have $\mathcal{O}_{4} \subset \mathcal{O}_{2}, \mathcal{O}_{1} \subset \mathcal{O}_{3}$, and $\mathcal{O}_{1} \cup \mathcal{O}_{2}=\mathcal{O}_{3} \cup \mathcal{O}_{4}$. This yields the three possibilities:

(a) $\mathbf{v} \in \mathcal{O}_{2} \cap \mathcal{O}_{3}: \mathbf{v}_{3}=\mathbf{v}_{2}, \mathbf{v}_{1}=\mathbf{v}$.

(b) $\mathbf{v} \in \mathcal{O}_{2} \cap \mathcal{O}_{4}: \mathbf{v}_{2}=\mathbf{v}_{1}=\mathbf{v}$.

(c) $\mathbf{v} \in \mathcal{O}_{3} \cap \mathcal{O}_{1}: \mathbf{v}_{3}=\mathbf{v}_{2}=\mathbf{v}_{1}$. 
By similar arguments to the case $n>0$, we conclude that $T_{J}$ is piecewise linear with exactly two pieces.

Now let $J=\mathbb{R}^{+}\left[\mathbf{v}, \mathbf{v}^{\prime}\right)$ be a sector of the type above. The first return map $T_{J}$ is piecewise linear on the sector with exactly two pieces, and we let $\mathbf{v}^{*}$ denote the breakpoint in the interior of $J$ (so $\mathbf{v}^{*}=\mathbf{v}_{3}$.) Let $M_{1}$ be the linear transformation corresponding to $J_{1}=\mathbb{R}^{+}\left[\mathbf{v}, \mathbf{v}^{*}\right.$ ), and let $M_{2}$ be the linear transformation corresponding to $J_{2}=\mathbb{R}^{+}\left[\mathbf{v}^{*}, \mathbf{v}^{\prime}\right)$, so that

$$
T_{J}(\mathbf{w})=\left\{\begin{array}{ll}
M_{1} \mathbf{w} & \text { if } \mathbf{w} \in \mathbb{R}^{+}\left[\mathbf{v}, \mathbf{v}^{*}\right) \\
M_{2} \mathbf{w} & \text { if } \mathbf{w} \in \mathbb{R}^{+}\left[\mathbf{v}^{*}, \mathbf{v}^{\prime}\right)
\end{array} .\right.
$$

Note that $M_{2} \mathbf{v}^{*}=\mathbf{v}$, and, by continuity, $M_{1} \mathbf{v}^{*}=\mathbf{v}^{\prime}$. Now, consider the action of $T_{J}^{(2)}$. In some neighborhood of $\mathbf{v}^{*}, T_{J}^{(2)}$ acts as $M_{2} M_{1}$ on points clockwise of $\mathbf{v}^{*}$ and as $M_{1} M_{2}$ on points counterclockwise of $\mathbf{v}^{*}$. If we can show that $T_{J}^{(2)}$ is linear on this neighborhood, then $M_{1} M_{2}=M_{2} M_{1}$ and we are done.

To compute $T_{J}^{(2)}$, one multiplies a sequence of matrices of the form $\left[\begin{array}{cc}c & -1 \\ 1 & 0\end{array}\right]$, with $c=a$ or $c=b$ at step $i$ if the $i$ th $x$-coordinate is positive or negative, respectively. Now, as we pass through $\mathbf{v}^{*}$, the sequence will change only at the two steps $j=j_{1}, j_{2}$ where $T_{a b}^{(j)}\left(\mathbf{v}^{*}\right)=(0, \pm 1)$. Furthermore, away from $\mathbf{v}^{*}$ in the neighborhood, if $c=a$ at one of those two steps, we must have $c=b$ at the other. Thus we find that (say)

$$
M_{1} M_{2}=M_{A}\left[\begin{array}{cc}
b & -1 \\
1 & 0
\end{array}\right] M\left[\begin{array}{cc}
a-1 \\
1 & 0
\end{array}\right] M_{B}
$$

and

$$
M_{2} M_{1}=M_{A}\left[\begin{array}{cc}
a & -1 \\
1 & 0
\end{array}\right] M\left[\begin{array}{cc}
b & -1 \\
1 & 0
\end{array}\right] M_{B},
$$

for certain matrices $M_{A}, M_{B}$ and $M$, in which $\tilde{M}:=M\left[\begin{array}{cc}*-1 \\ 1 & 0\end{array}\right]$ is a product of matrices corresponding to the sequence of iterations between the two places where $T_{a b}^{(j)}\left(\mathbf{v}^{*}\right)=(0, \pm 1)$. Note that the value of $*(a$ or $b)$ has no effect on the first step of the iteration of $T_{a b}$. The matrix equation $M_{1} M_{2}=M_{2} M_{1}$ boils down to showing that

$$
\left[\begin{array}{cc}
a-1 \\
1 & 0
\end{array}\right] M\left[\begin{array}{cc}
b & -1 \\
1 & 0
\end{array}\right]=\left[\begin{array}{cc}
b & -1 \\
1 & 0
\end{array}\right] M\left[\begin{array}{cc}
a-1 \\
1 & 0
\end{array}\right] .
$$

We now recall from Theorem [3.2(ii), the key fact that if $S_{a b}^{(n)}(0,1)=(0,-1)$ for some (positive or negative) integer $n$, then

$$
T_{a b}^{(n)}(0,1)=(0,-1) .
$$

If $n>0$, this gives $\tilde{M}(0,1)=M(-1,0)=(0,-1)$; if $n<0$ we obtain instead $\tilde{M}(0,-1)=$ $M(1,0)=(0,1)$. In either case, we can conclude that

$$
M=\left[\begin{array}{cc}
0 & -1 \\
1 & d
\end{array}\right]
$$

for some value $d$, and it follows that

$$
\left[\begin{array}{cc}
a & -1 \\
1 & 0
\end{array}\right] M\left[\begin{array}{cc}
b-1 \\
1 & 0
\end{array}\right]=\left[\begin{array}{cc}
-a-b-d & 1 \\
-1 & 0
\end{array}\right],
$$


This is symmetric in $a$ and $b$, hence $M_{1} M_{2}=M_{2} M_{1}$, and the theorem is proved.

In preparation for the next result, we recall the following well-known fact, see Arnold and Avez [1, Appendix 27]. .

Lemma 3.1 Let $M=\left[\begin{array}{ll}a & b \\ c & d\end{array}\right] \in S L(2, \mathbb{R})$, and suppose that $M \neq \pm I$. Then the map $(x, y) \rightarrow$ $M(x, y)$ leaves invariant the quadratic form

$$
Q(x, y)=c x^{2}+(d-a) x y-b y^{2},
$$

and the only quadratic forms it leaves invariant are scalar multiples of $Q(x, y)$. The level sets $c x^{2}+(d-a) x y-b y^{2}=\lambda$ for real $\lambda$ are invariant sets, and fill the plane. Furthermore:

(1) If $|\operatorname{Tr}(M)|<2$, then the nonempty level sets are ellipses, or else a single point.

(2) If $|\operatorname{Tr}(M)|>2$, then the level sets are hyperbolas, except for $\lambda=0$, where they are their asymptotes, consisting of two straight lines through the origin.

(3) If $|\operatorname{Tr}(M)|=2$, then the nonempty level sets are either two parallel lines whose vector sum is a fixed parallel line through the origin; or, for $\lambda=0$, this fixed line.

Any matrix $M^{\prime}$ commuting with $M$ also preserves the quadratic form $Q$.

Proof. The condition for invariance of the quadratic form $Q(x, y)=A x^{2}+2 B x y+C y^{2}$ is that $M^{T} Q M=Q$, where $Q=\left[\begin{array}{ll}A & B \\ B & C\end{array}\right]$; an equivalent matrix condition is that $M^{T} Q=Q M^{-1}$. Letting $M=\left[\begin{array}{ll}a & b \\ c & d\end{array}\right] \in S L_{2}(\mathbb{R})$, the latter condition yields the linear equations $(a-d) A=-2 c B$, $c C=-b A$, and $(a-d) C=2 b B$. At least one of $b, c, a-d$ is nonzero, otherwise $M= \pm I$ which we have excluded. Then this linear system has rank at least two, so has at most a oneparameter family of solutions. For $\operatorname{det}(M)=1$ (and $M \neq \pm I$ ) the system has rank two and has the one-parameter family of solutions $(A, 2 B, C)=\alpha(c, d-a,-b)$ for $\alpha \in \mathbb{R}$. Choosing $\alpha=1$, its discriminant is

$$
\operatorname{Disc}(Q):=(d-a)^{2}+4 b c=\operatorname{Tr}(M)^{2}-4 .
$$

Cases (1)-(3) correspond to the discriminant being negative, positive or zero.

Diagonalizing $M$ over the complexe numbers gives either $\left[\begin{array}{ll}x & 0 \\ 0 & x^{-1}\end{array}\right]$ or $\pm\left[\begin{array}{ll}1 & x \\ 0 & 1\end{array}\right]$; in either case, we find that any matrix $M^{\prime}$ commuting with $M$ is of the form $M^{\prime}=e M+f I$. Substituting this in, we find that $M^{\prime}$ also preserves $Q$.

Theorem 3.4 Suppose that $S_{a b}$ has irrational rotation number and that the $S_{a b}$ orbit of $(0,1)$ contains $(0,-1)$. Then the $T_{a b}$ orbit of $(0,1)$ contains $(0,-1)$, and $T_{a b}$ has a piecewise conic invariant circle. If $S_{a b}^{(n)}(0,1)=(0,-1)$ for $n \in \mathbb{Z}$, then the number of conic pieces of the invariant circle is at most $|n|$, and all conic pieces are of the same type, either arcs of ellipses, arcs of hyperbolas or line segments, respectively.

Proof. The fact that the $T_{a b}$ orbit of $(0,1)$ contains $(0,-1)$ follows from Theorem 3.2 since irrational rotation number can only occur in case (ii).

Let $\mathcal{C}$ be the closure of the orbit containing $\mathbf{v}_{0}:=(0,1)$; we prove below that $\mathcal{C}$ is an invariant circle with the required properties. If so, it follows that all $T_{a b}$ orbits are scaled copies of this invariant circle. 
By hypothesis $S_{a b}^{(n)}(0,1)=(0,-1)$ with $n \in \mathbb{Z}$; the value of $n$ is unique since $S_{a b}$ has irrational rotation number. Set $\mathcal{O}=\left\{S_{a b}^{(i)}(0,1): 0<i \leq n\right\}$ if $n>0$ and set $\mathcal{O}=\left\{S_{a b}^{(i)}(0,1)\right.$ : $n<i \leq 0\}$ if $n<0$; this is the minimal set $\mathcal{O}$ to which Theorem 3.3 applies. It follows that the rays determined by the points of $\mathcal{O}$ partition of the plane into $|n|$ sectors, and on each sector the first return map of $T_{\mu \nu}$ is piecewise linear, with two commuting pieces $M_{1}, M_{2}$.

We consider the sector $J=\mathbb{R}^{+}\left[\mathbf{v}, \mathbf{v}^{\prime}\right)$ in which $\mathbf{v}$ is whichever of $(0,1)$ or $(0,-1)$ occurs first in $\mathcal{O}$. We have each $M_{j} \neq \pm I$ because, if not, then the rotation map $S_{a b}$ would have a periodic point, and hence a rational rotation number, a contradiction. Now Lemma 3.1 applies, and shows that $M_{1}$ and $M_{2}$ each leave invariant a one-parameter family of quadratic forms, multiples of $Q_{1}$ and $Q_{2}$, respectively; that is, $M_{1}^{T} Q_{1} M_{1}=Q_{1}$ and $M_{2}^{T} Q_{2} M_{2}=Q_{2}$. Moreover, since $M_{1}$ and $M_{2}$ commute, we can take $Q_{1}=Q_{2}$.

The claim shows that all the return visits of $\mathbf{v}$ to $J$ lie on a single level set of the quadratic form $Q_{1}$, with the level set parameter $\lambda$ specified by requiring that $\mathbf{v}$ lie on this conic. Since $S_{a b}$ has irrational rotation number the return visits are dense on that part of this conic section in the sector $J$, and the closure is the full arc of the conic section, call it $\mathcal{C}_{1}$, that intersects the sector. In the hyperbola case $\left|\operatorname{Tr}\left(M_{1}\right)\right|>2$, the sector $J$ cannot include an eigenvector of $M_{1}$ (an asymptote), because if it did, then the map $S_{a b}$ would have a periodic point, and hence a rational rotation number, a contradiction; thus the sector $J$ contains an arc of one connected component of a hyperbola.

The $\operatorname{arc} \mathcal{C}_{1}$ is either an arc of an ellipse or a hyperbola, or a line segment. By similar reasoning, on each other sector $J_{i}$, the visits of the orbit of $\mathbf{v}_{0}$ fill out the arc of a unique conic on that sector. The image of the $\operatorname{arc} \mathcal{C}_{1}$, when iterated under $T_{a b}$ will visit each sector; this visit is given by a linear map, so the conic $\mathcal{C}_{i}$ is the same type of conic (linear, ellipse, or hyperbola) as $\mathcal{C}_{1}$.

It remains to show that the union $\mathcal{C}=\cup_{j=1}^{n} \mathcal{C}_{j}$ of these pieces is a connected set. To see this, consider the preimage $\mathbf{v}_{-1}=T_{a b}^{-1}(\mathbf{v})$. This point is not in $\mathcal{O}$, so it necessarily lies in the interior of some sector $J_{i}$, hence is contained in the conic piece $\mathcal{C}_{i}$. Now, the forward iteration $T_{a b}\left(\mathcal{C}_{i}\right)$ will cover the point $\mathbf{v}_{0}$ in its interior. This image is a conncected set, so the conic pieces $\mathcal{C}$ and $\mathcal{C}_{j}$ in the sector $J_{j}$ adjacent to $J$, moving clockwise, must touch at the point $\mathbf{v}_{0}$. Next, forward iteration of $T_{a b}$ of a connected neighborhood of $\mathbf{v}$ over the full set $\mathcal{O}$ shows that all segments $\mathcal{C}_{j}$ glue together into a connected set. Thus $\mathcal{C}$ is an invariant circle.

Remark. The conclusion of Theorem 3.4 need not hold without the requirement of irrational rotation number, see Example 4.3 in $\S 4$.

\section{Examples}

In this section we first give examples of one-parameter families where $(0,1)$ and $(0,-1)$ are in the same orbit, which illustrate the theorems in $\S 3$.

Example 4.1 If $1<a<\sqrt{2}$, and $b=2 \frac{a^{2}-1}{a\left(a^{2}-2\right)}$, then $T_{a b}$ is either periodic or has a piecewise

elliptical invariant circle. (These parameter values come from assuming that $T_{a b}^{(8)}(0,-1)=$ $(0,1)$, with appropriate sign conditions.) 
A detailed analysis of this example is given in the appendix, where the following facts are proved. One chooses $\mathcal{O}=\left\{T_{a b}^{(j)}: 1 \leq j \leq 8\right\}$. When the rotation number is rational, $T_{\mu \nu}$ is periodic; when it is irrational Theorem 3.4 applies to show that it has a piecewise conic invariant circle with at most eight pieces. The conic sections are always ellipses.

There is an explicit formula for the rotation number. Set $a=2 \cos \theta$, with $\pi / 4<\theta<\pi / 3$. The rotation number $r\left(S_{a b}\right)$ is explicitly given by

$$
r\left(S_{a b}\right)=\frac{3 \pi-7 \theta}{14 \pi-32 \theta} .
$$

It follows that $r\left(S_{a b}\right)$ is rational if and only if $\theta$ is a rational multiple of $\pi$.

The special case $\nu=0, \mu=\sqrt[4]{2}$ (corresponding to $a=-b=\sqrt[4]{2}$ ) has a rotation number which is provably irrational. Indeed, one checks that $z=e^{i \theta}$ is a root of $z^{8}+4 z^{6}+4 z^{4}+4 z^{2}+1=$ 0 ; this equation has no roots of unity as roots, so $\theta$ is not a rational multiple of $\pi$. An invariant circle of $T_{\mu \nu}$ is pictured in Figure 4.1] it is a piecewise union of eight arcs of ellipses. (In this case several of the arcs are parts of a single ellipse.)

A related example comes from example 3.2 in part I, which has parameters $a=\cos \frac{2 \pi}{n}$ and $b=2 \cos \theta$ with $0<\theta<\pi$ for $\theta$ an irrational multiple of $\pi$, then $r\left(S_{a b}\right)=\frac{\theta}{\pi+n \theta}$ is irrational, and this case has piecewise elliptic invariant circles.

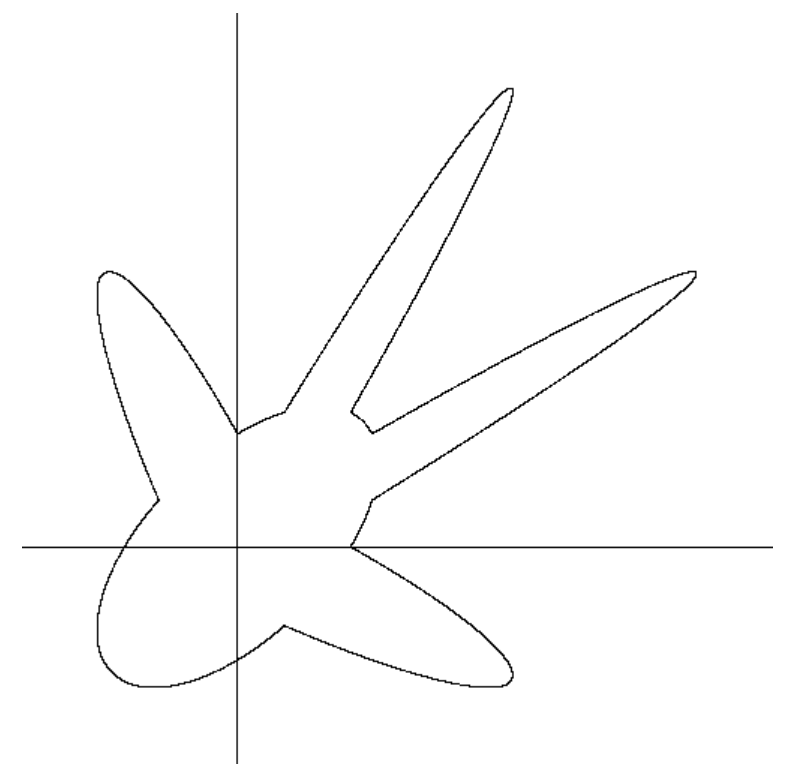

Figure 4.1: Invariant circle for $a=\sqrt[4]{2}, b=-\sqrt[4]{2}$ ("Bugs Bunny").

Example 4.2 If $0<a<1$, and $b=\frac{1}{2} \frac{3 a^{2}-3+\sqrt{a^{4}-2 a^{2}+9}}{a\left(a^{2}-2\right)}$, then $T_{a b}$ is either periodic or has a piecewise conic invariant circle. All three cases of piecewise ellipses, straight lines or hyperbolas occur, depending on the parameter value. (These parameter values come from assuming that $T_{a b}^{(10)}(0,1)=(0,-1)$, with appropriate sign conditions.)

A detailed analysis of this example is given in the appendix, where the following facts are proved. We take $\mathcal{O}=\left\{T_{a b}^{(j)}(0,1): 1 \leq j \leq 10\right\}$. In the case of rational rotation number 
the map $T_{a b}$ is periodic. For irrational rotation number Theorem 3.4 applies, and there are three cases, depending on the trace of $M_{1}$. One has $0<\operatorname{Tr}\left(M_{1}\right)<2$ for $1<a<\alpha_{0}$ where $\alpha_{0} \approx 0.3802775690976 \ldots$ is the real root of $x^{4}+3 x^{3}+3 x^{2}+x-1$ in the unit interval; invariant circles are then piecewise arcs of ellipses. One has $\operatorname{Tr}\left(M_{1}\right)=2$ for $a=\alpha_{0}$; the invariant circle in this case is piecewise linear. One has $\operatorname{Tr}\left(M_{1}\right)>2$ for $\alpha_{0}<a<1$; invariant circles are then piecewise arcs of hyperbolas.

The following three figures picture invariant circles for cases of Example 4.2, Figure 4.2 pictures the case $\left.a=\sqrt{\frac{\sqrt{5}-1}{2}}\right) \approx 0.78615$, which is the case $b=-a$. The rotation number is irrational (in fact, transcendental) and the invariant circle consists of ten arcs of hyperbolas.

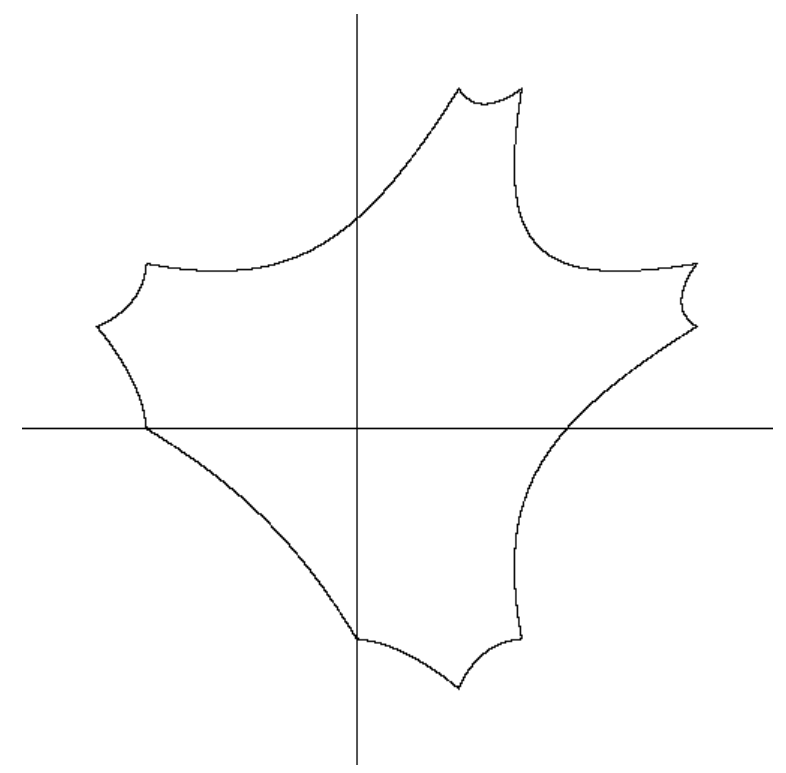

Figure 4.2: Invariant circle for $a=\left(\frac{\sqrt{5}-1}{2}\right)^{1 / 2}, b=-\left(\frac{\sqrt{5}-1}{2}\right)^{1 / 2}$ ("Elmer Fudd").

Figure 4.3 pictures the case of the critical value $a=\alpha_{0} \approx 0.3802$. The rotation number

$$
r\left(T_{\mu \nu}\right)=\frac{2 \alpha_{0}^{2}+1}{9 \alpha_{0}^{2}+4}
$$

is an irrational algebraic number. Theorem 3.4 applies to show it has an invariant circle, which is piecewise linear with ten pieces.

Figure 4.4 pictures the case $\alpha=1 / 10$. In this case the rotation number is irrational and the invariant circle consists of ten arcs of ellipses.

Example 4.3 If $1<a<\sqrt{2}$, and $b=\frac{(a-1)\left(2 a^{3}-4 a-1\right)}{a\left(a^{2}-2\right)\left(a^{2}-a-1\right)}$, then $T_{a b}^{(13)}(0,-1)=(0,1)$, but $S_{a b}$ has constant rational rotation number $r\left(S_{a b}\right)=\frac{1}{5}$ and $T_{a b}$ has no invariant circles. In this case all orbits diverge.

The range of $b$ is $-\infty<b<0$. Theorem 3.3 and 3.4 do not apply, because all $S_{a b}$ in this family have constant rational rotation number $r=\frac{1}{5}$. The following facts are proved in the 


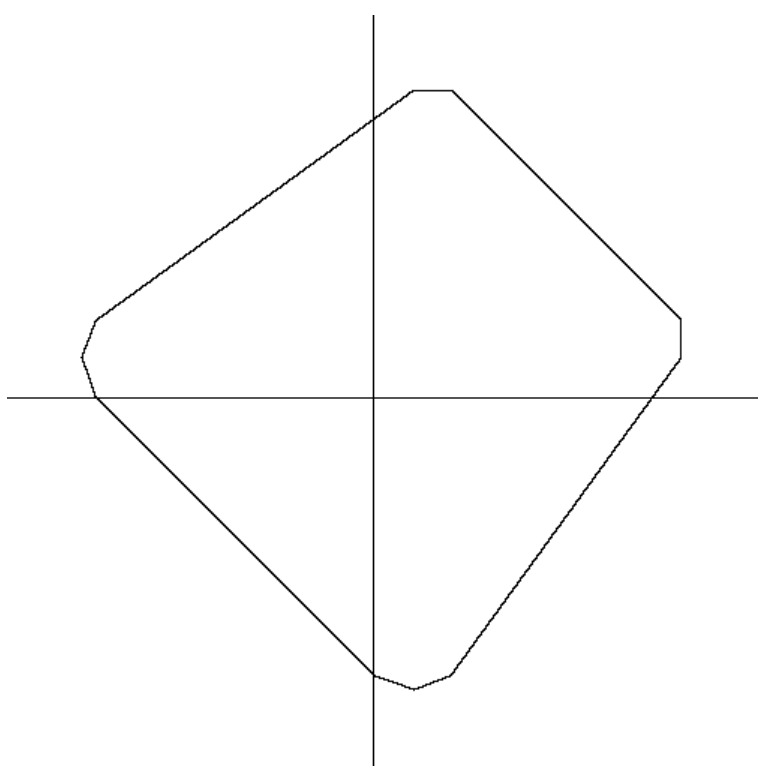

Figure 4.3: Piecewise linear invariant circle for critical value $a=\alpha_{0}=0.380277, b$ as in example 4.2

appendix. If we take $\mathcal{O}=\left\{T^{(j)}(0,-1): 1 \leq j \leq 13\right\}$ then part of the conclusion of Theorem 3.3 still applies: there exists a sector $J=\mathbb{R}^{+}\left[\mathbf{v}, \mathbf{v}^{\prime}\right)$ given by two elements of $\mathcal{O}$ which has a well defined first return map, which consists of exactly two pieces, and the resulting matrices $M_{1}$ and $M_{2}$ commute. (However not all sectors have a well-defined first return map.) For the sector $J$ the matrices $M_{j}$ have $\left|\operatorname{Tr}\left(M_{j}\right)\right|>2$, so their invariant sets restricted to $J$ are hyperbolic arcs; however the asymptotes of the hyperbolas also lie in $J$. These maps fall under case (ii) of Theorem 2.1 all orbits are unbounded. Each $T_{a b}$ in this family satisfy the following weak form of the invariant circle property: Each orbit of $T_{a b}$ either falls on a finite number of rays, or else lies on a finite union of (unbounded) hyperbolic arcs.

A specific example is the parameter values $a=-b \approx 1.235877977$ where $a$ is a root of $x^{6}-x^{5}-x^{4}-2 x^{2}+3 x+1=0$.

It appears plausible that there are many more parameter values having invariant circles. We present several non-rigorous numerical examples showing numerical plots of orbits for other parameter values; these are intended to illustrate the structure of (possible) general invariant circles. The parameter values exhibited here were found by iterative trial-and-error search, in which the first 10000 iterates appeared to form an invariant circle. These include the values $(a, b)$ used in making the Figures 4.54 .9 below. One should view these figures with a grain of salt; a sufficiently slowly divergent periodic orbit could produce a picture that looked like an invariant circle; qualitatively, the longer the period of the periodic orbit, the closer the corresponding eigenvalue seems to tend to be to 1 , so an orbit with very high period will tend to diverge very slowly, and will, furthermore, look like a curve. However Conjecture A together with various theorems in $\S 2$ would imply that the set of parameter values having an irrational rotation number has positive two-dimensional Lebesgue measure. If so, this makes it plausible that the computer plots actually approximate invariant circles. That is, even if 


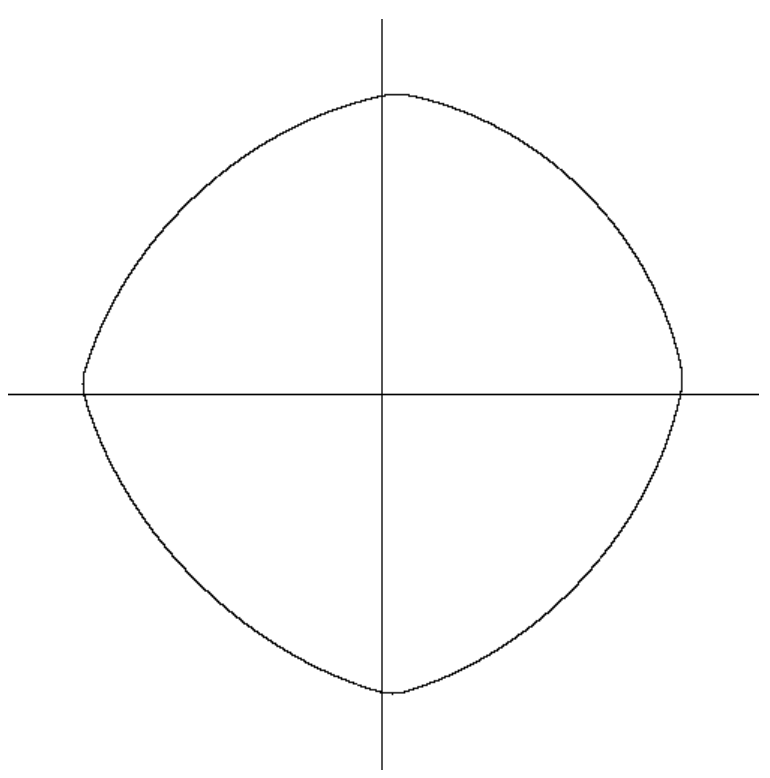

Figure 4.4: Invariant circle for $a=0.1, b$ as in example 4.2 .

the parameter values below don't correspond to invariant circles (as seems likely), then very small parameter changes should give some $T_{a b}$ having an invariant circle exhibiting the same qualitative appearance. One may compare the apparant invariant circle in Figure 4.9 with one pictured in Froeschlé [4, which stimulated the work of Herman [6. Chap. VIII] on existence of invariant circles for these maps.

Example $4.4 a=0.2, b=-0.7$. (Figure 4.5) At this scale the invariant circle appears to be smooth; it seems unlikely that this is actually the case.

Example 4.5 $a=1.4, b=-1.4$. (Figure 4.6) This highlights a typical behavior of the generic invariant circle; there are oscillations on several frequency scales (the thickness of the curve results from oscillations of wavelength smaller than the pixel size). The "hair" is usually not as pronounced as this.

Example 4.6 $a=-.9, b=-4$. (Figure 4.7) The long spikes in the second and fourth quadrants are typical of invariant circles when $a, b<0$, although, again, not usually as pronounced, for larger values of $b$.

Example $4.7 a=1.5, b=1.1$. (Figure 4.8) For $a, b>0$, on the other hand, the first and third quadrants features tend to be pronounced.

Example $4.8 a=1.9, b=-.2$. (Figure 4.9) For $a>0>b$, the first quadrant alone tends to be pronounced if $a>>-b$ (otherwise, as in figure 4.5), no quadrant seems to dominate). 


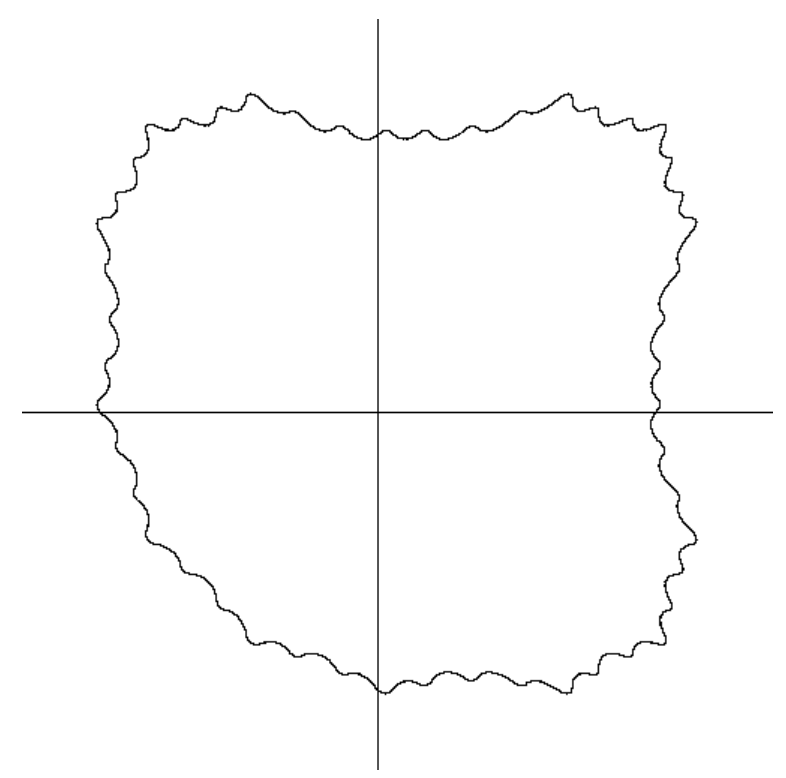

Figure 4.5: Apparent invariant circle for $a=0.2, b=-0.7$.

\section{The Invariant Conic Set $\Omega_{Q}$ and $\Omega_{S B}$}

We defined $\Omega_{Q}$ to be the set of parameter values $(a, b)$ for which $T_{a b}$ has a piecewise conic invariant circle of the type given in Theorem 3.3 . i.e. parameter values such that the $S_{a b}$-orbit of $(0,1)$ contains $(0,-1)$, and $S_{a b}$ has irrational rotation number. The fact that that the orbit of $(0,1)$ is restricted makes this a rather small set.

Theorem 5.1 The set $\Omega_{P} \cup \Omega_{Q}$ has Hausdorff dimension 1 .

Proof. We have exhibited parametric curves in this set in the examples, so it remains to show that $\Omega_{P}$ and $\Omega_{Q}$ each have Hausdorff dimension at most one.

We treat $\Omega_{Q}$, the argument for $\Omega_{P}$ being similar. Theorem 3.3 gives the necessary condition that either $S_{a b}^{(k)}(0,1)=(0,-1)$ or $S_{a b}^{(k)}(0,-1)=S_{a b}(0,1)$, for some $k \in \mathbb{Z}_{>0}$. These conditions (namely $T_{a b}^{(k)}(0,1)_{x}=0$, subject to the constraint $T_{a b}^{(k)}(0,1)_{y}<0$, for each $k \in \mathbb{Z} \backslash\{0\}$ ) cut out a countable number of pieces of real algebraic curves. The resulting set contains $\Omega_{Q}$. It follows that $\Omega_{Q}$ has Hausdorff dimension at most 1 .

In the introduction we proposed the following conjecture.

Conjecture A. The set $\Omega_{P} \cup \Omega_{Q}$ is a dense subset of $\Omega_{S B}$.

We give some justification for this conjecture. For any positive $\epsilon$, if $r\left(S_{\mu \nu}\right)$ is irrational, there is a perturbation of $\nu$ smaller than $\epsilon$ such that $T_{\mu \nu^{\prime}}$ maps $(0,1)$ to $(0,-1)$; that is, the perturbation goes to $(0,-\lambda)$ for some positive $\lambda$ and by Theorem 3.2 (ii) of part I, it must be the case that $\lambda=-1$. One can rigorously show, for any rational, there is such a point having rotation number between that rational and $r\left(S_{\mu \nu}\right)$. Experimentation suggests that this point will usually be in $\Omega_{P} \cup \Omega_{Q}$, but the example 4.3 shows one way this can fail. 


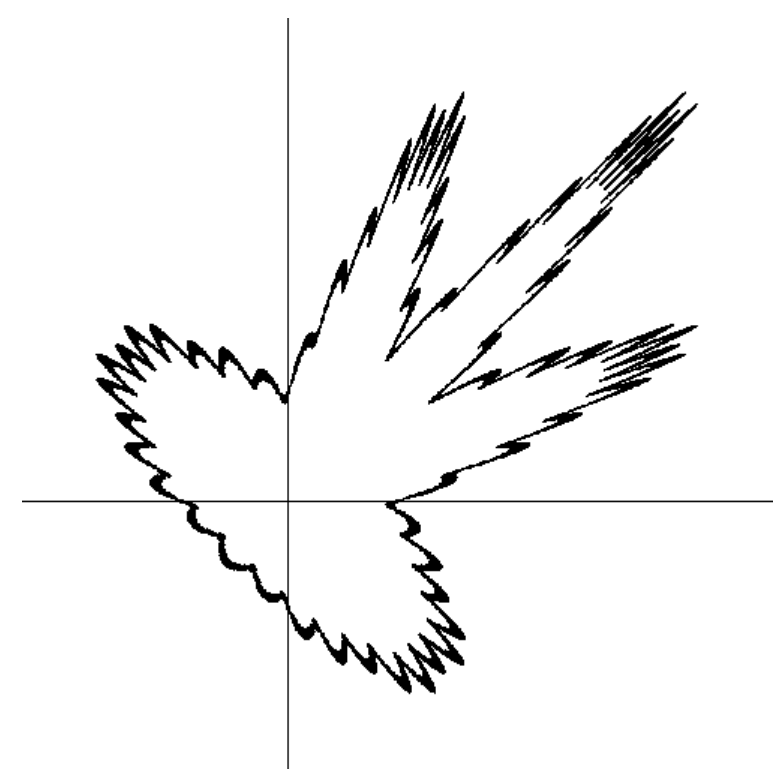

Figure 4.6: Apparent invariant circle for $a=1.4, b=-1.4$.

As an example, we consider the problem of finding points of $\Omega_{\text {per }} \cup \Omega_{\text {conic }}$ close to the parameter values $(a, b)=(1.4,-1.4)$ which has the (apparent) invariant circle pictured in Figure 4.6. We found numerically that

$$
a=-b=1.399999999996869855138895062694261779494
$$

has a piecewise elliptic invariant circle (or is periodic), with $T^{(810096)}(0,-1)=(0,1)$, complex eigenvalues. We also found numerically that

$$
a=-b=1.400000000000000366642660387254241957986
$$

has a piecewise hyperbolic invariant circle (or is periodic), with $T^{(35676178)}(0,1)=(0,-1)$, no eigenvectors in interval.

Both these parameter sets produce plots basically indistinguishable from Figure 4.6. Since the simpler case has 810096 conic pieces, the piecewise conic structure is invisible in the computer plot.

In fact, $\Omega_{P} \cup \Omega_{Q}$ is contained in a countable union of real algebraic curves; the argument above would say that any 2 -dimensional ball centered at a point of $\Omega_{S B}$ would contain a segment of such a curve. In nearly all cases, as exhibited in Examples 4.1 and 4.2 it appears that such a curve does not have constant rotation number; if so, then it necessarily contains a dense set of points with irrational rotation number, and one can guarantee a point of $\Omega_{\text {conic }}$ in any neighborhood, so that Conjecture A would follow.

To conclude, we remark on the general question of the existence of invariant circles when $r\left(S_{a b}\right)$ has irrational rotation number. A heuristic from KAM theory [9] is that if the rotation number has very good rational approximations, then the orbit has the possiblity to diverge, since it is very close for a long time to a number of divergent parameter values. On the other hand, if the rotation number $r$ is irrational, then the $S_{\mu \nu}$ orbit of $(0,1)$ will approach arbitrarily 


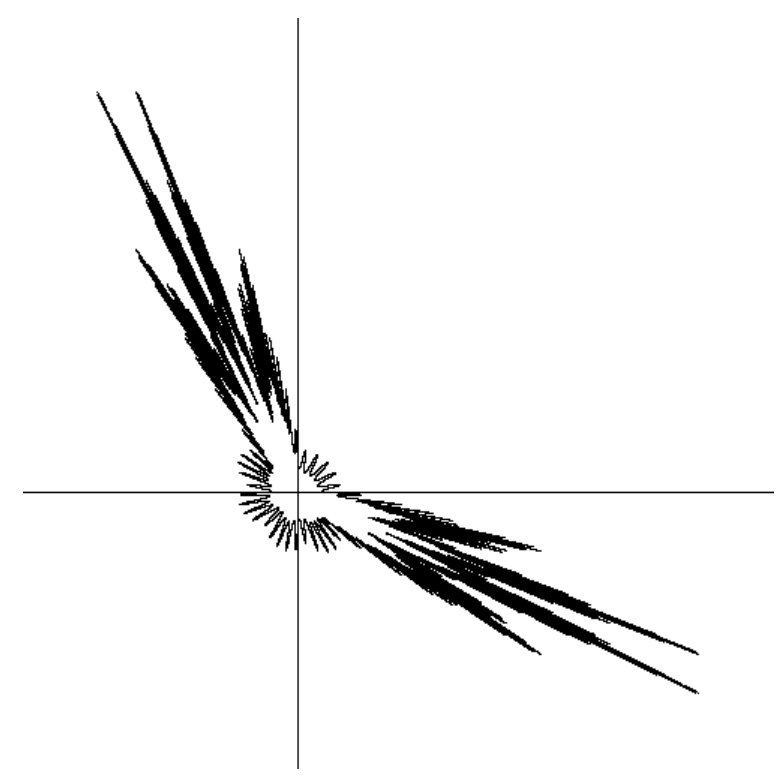

Figure 4.7: Apparent invariant circle for $a=-0.9, b=-4$.

close to $(0,-1)$ infinitely often; this ought to place some constraints on the extent to which the size of the iterates of $(0,1)$ can vary, and suggests the heuristic that under some Diophantine condition on $r$ this might lead to an invariant circle. Indeed the result of Herman 66, VIII.5.2] shows this is the case when the rotation number is of constant type (i.e. a badly approximable number). It remains an open problem to decide if there exist parameters $(a, b)$ for which $S_{a b}$ has irrational rotation number and $T_{a b}$ has no invariant circle. The property of having no invariant circle, if it occurs, will not come from a Diophantine property alone of the rotation number $r\left(S_{a b}\right)$; the particular parameter values $(a, b)$ will matter. This is indicated by Example 4.1. which gives a family with continuously varying rotation number in which all parameter values having irrational rotation number necessarily have an invariant circle; all parameter values in some open interval occur. It might be helpful in resolving this open problem to better understand the mechanism by which these examples, which can have an irrational rotation number with arbitrary Diophantine properties, circumvent the KAM heuristic above. 


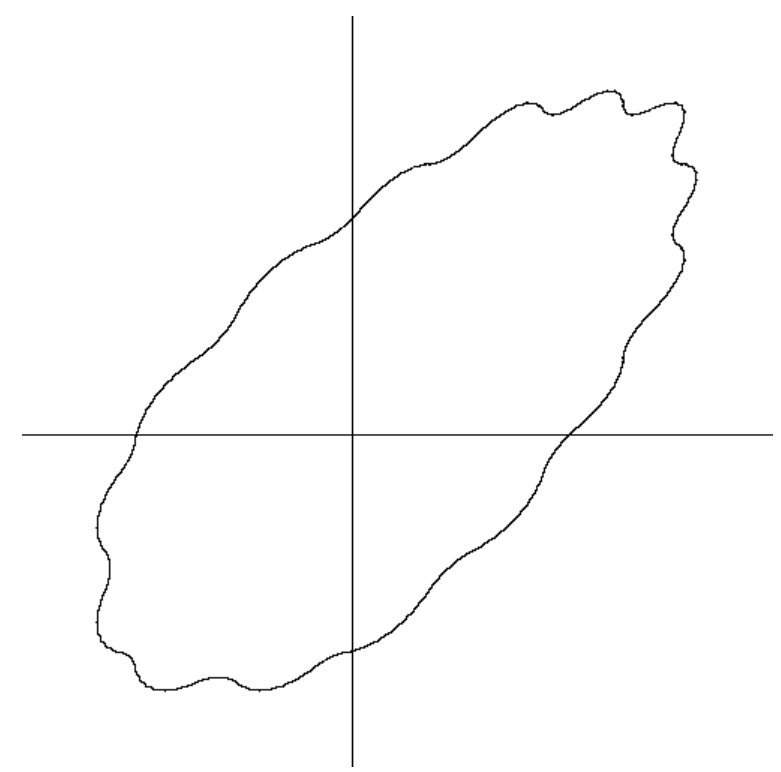

Figure 4.8: Apparent invariant circle for $a=1.5, b=1.1$.

\section{Appendix: Rigorous Verification of Examples}

In this appendix we give proofs for the assertions made in $\S 5$ about Examples 4.1 4 4.3. The calculations for Example 4.1 below can be verified by hand, but those for Example 4.2 and 4.3 require a computer. The packages MAPLE and MAGMA were used for some of the calculations below.

Example 4.1. $T_{a b}$ with $1<a<\sqrt{2}$, with $b=2 \frac{a^{2}-1}{a^{3}-2 a}$. Here $b \in(-\infty, 0)$, and $T_{a b}^{(8)}(0,-1)=$ $(0,1)$.

Proof. We first verify that one has $T_{a b}^{(8)}(0,-1)=(0,1)$. Set $\mathbf{v}_{0}=(0,-1)$ and $\mathbf{v}_{j}=T_{a b}^{(j)}\left(\mathbf{v}_{0}\right)$; the sequence of signs $\left.S_{j}=\operatorname{Sign}\left(\mathbf{v}_{j}\right)_{x}\right)$ determine the sequence of iterates. The resulting $\mathbf{v}_{j}$ and their sign values are given in Table 6.1]

\begin{tabular}{|l|r|}
\hline \multicolumn{1}{|c|}{ vector } & $\left.\operatorname{Sign}\left(\mathbf{v}_{j}\right)_{x}\right)$ \\
\hline $\mathbf{v}_{1}=(1,0)$ & + \\
$\mathbf{v}_{2}=(a, 1)$ & + \\
$\mathbf{v}_{3}=\left(a^{2}-1, a\right)$ & + \\
$\mathbf{v}_{4}=\left(a^{3}-2 a, a^{2}-1\right)$ & - \\
$\mathbf{v}_{5}=\left(a^{2}-1, a^{3}-2 a\right)$ & + \\
$\mathbf{v}_{6}=\left(a, a^{2}-1\right)$ & + \\
$\mathbf{v}_{7}=(1, a)$ & + \\
$\mathbf{v}_{8}=(0,1)$ & \pm \\
\hline
\end{tabular}

Table 6.1: Orbit $\mathcal{O}$ for example 4.1 


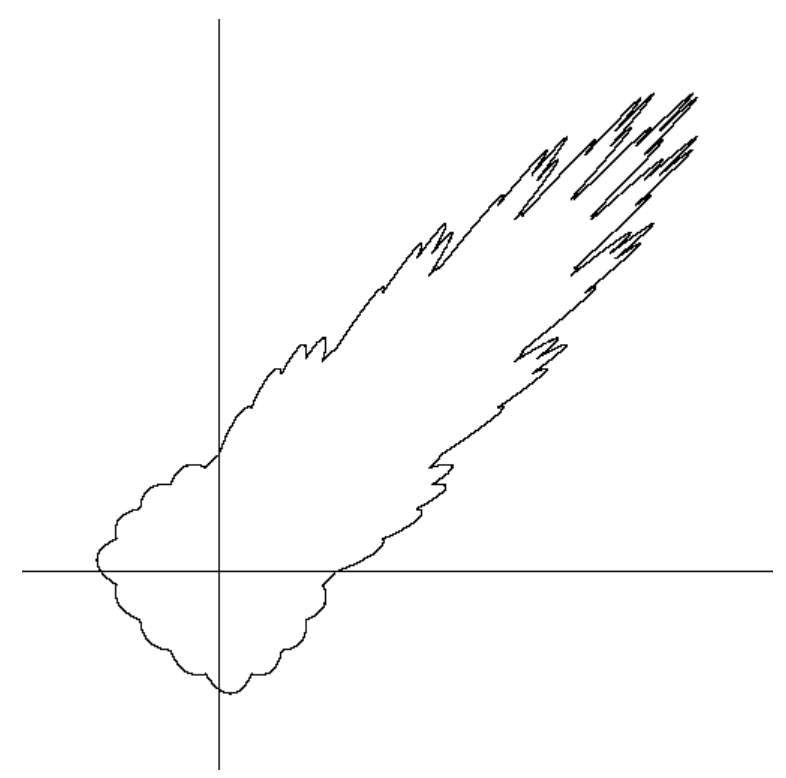

Figure 4.9: Apparent invariant circle for $a=1.9, b=-0.2$.

To verify the entries in Table 6.1 note that the condition $1<a<\sqrt{2}$ determines $\left\{\mathbf{v}_{j}: 1 \leq\right.$ $j \leq 4\}$ and their signs, and in particular

$$
\mathbf{v}_{5}=\left(\left(a^{3}-2 a\right) b+1-a^{2}, a^{3}-2 a .\right)
$$

We impose the condition that $\mathbf{v}_{5}=R\left(\mathbf{v}_{4}\right)$, and this requires that

$$
\left(a^{3}-2 a\right) b+2-2 a^{2}=0
$$

which specifies $b$ uniquely. Now the relation $T_{a b}^{-1}=R \circ T_{a b} \circ R^{-1}$ yields $\mathbf{v}_{j}=R\left(\mathbf{v}_{9-j}\right)$ for $5 \leq j \leq 8$, and also determines their symbol sequence. This completes Table 6.1] and shows $T_{a b}^{8}(0,-1)=(0,1)$.

We choose $\mathcal{O}=\left\{\mathbf{v}_{j}: 1 \leq j \leq 8\right\}$, and the hypotheses of Theorem 3.3 and 3.4 apply whenever the rotation number $r\left(S_{a b}\right)$ is irrational. The elements of $\mathcal{O}$ appear in counterclockwise angular order as

$$
\mathbf{v}_{1}, \mathbf{v}_{6}, \mathbf{v}_{2}, \mathbf{v}_{7}, \mathbf{v}_{3}, \mathbf{v}_{8}, \mathbf{v}_{4}, \mathbf{v}_{5}
$$

We choose the sector $J:=\mathbb{R}^{+}\left[\mathbf{v}, \mathbf{v}^{\prime}\right)=\mathbb{R}^{+}\left[\mathbf{v}_{4}, \mathbf{v}_{5}\right)$. This sector includes the entire third quadrant with signs $(-,-)$; here $\mathbf{v}_{4}$ and $\mathbf{v}_{5}$ have signs $(-,+)$ and $(+,-)$, respectively. The breakpoint for $J$ is the ray determined by

$$
\mathbf{v}^{*}:=(0,-1)
$$

The first return maps on the subsectors $J_{1}=\mathbb{R}^{+}\left[\mathbf{v}, \mathbf{v}^{*}\right)$ and $J_{2}=\mathbb{R}^{+}\left[\mathbf{v}^{*}, \mathbf{v}^{\prime}\right)$ are well-defined regardless of whether the rotation number is rational or irrational.

The first return map on $J_{1}$ is $T_{a b}^{(5)}$ and has symbol seqence $(-++++)$; its associated matrix ${ }^{1} M_{1}$ is:

$$
M_{1}=\left[\begin{array}{cc}
a & -a^{2}+1 \\
a^{2}-1 & -a^{3}+2 a
\end{array}\right]
$$

\footnotetext{
${ }^{1}$ To express the entries of $M_{1}$ in terms of the variable $a$ alone, the variable $b$ is eliminated using (6.1).
} 
The first return map on $J_{2}$ is $T_{a b}^{(4)}$ with symbol sequence $(++++)$ and its associated matrix $M_{2}$ is:

$$
M_{2}=\left[\begin{array}{cc}
a^{4}-3 a^{2}+1 & -a^{3}+2 a \\
a^{3}-2 a & -a^{2}+1
\end{array}\right]
$$

We have $\operatorname{Tr}\left(M_{1}\right)=-a^{3}+3 a$ so

$$
0<\operatorname{Tr}\left(M_{1}\right)<2 \quad \text { for } \quad 1<a<\sqrt{2} .
$$

We parametrize the eigenvalues and eigenvectors of $M_{1}$ and $M_{2}$ using the parameter $\theta$, with

$$
a=2 \cos \theta \quad \text { for } \quad \frac{\pi}{4}<\theta<\frac{\pi}{3} .
$$

The common eigenvectors $\mathbf{w}^{+}$and $\mathbf{w}^{-}$of $M_{1}$ and $M_{2}$ are given by

$$
\mathbf{w}^{ \pm}=\left(e^{ \pm i \theta}, 1\right)
$$

A calculation (using $a=e^{i \theta}+e^{-i \theta}$ ) gives

$$
\begin{gathered}
M_{1} \mathbf{w}_{+}=-e^{3 i \theta} \mathbf{w}^{+}, \quad M_{1} \mathbf{w}^{-}=-e^{3 i \theta} \mathbf{w}^{-} . \\
M_{2} \mathbf{w}^{+}=e^{4 i \theta} \mathbf{w}^{+}, \quad M_{2} \mathbf{w}^{-}=e^{-4 i \theta} \mathbf{w}^{-} .
\end{gathered}
$$

The matrices $M_{1}$ and $M_{2}$ leave invariant a common family of ellipses centered at the origin. Such a family of ellipses centered at the origin is specified by a single parameter $\phi$ with $0 \leq \phi<2 \pi$, and the family elements parametrized by $r>0$, with

$$
E=E(r, \phi):=\{\mathbf{v}(\omega)=(r \cos (\phi+\omega), r \cos (\omega)): 0 \leq \omega<2 \pi\} .
$$

The action of $M_{1}$ on that portion of the ellipse in $J$ is to take $\mathbf{v}(\omega)$ to $\mathbf{v}(\omega+\pi-3 \theta)$, while the action of $M_{2}$ there takes $\mathbf{v}(\omega)$ to $\mathbf{v}(\omega-2 \pi+4 \theta)$; the multiples of $\pi$ are specified to keep the image angle in $[0,2 \pi]$.

We now show that the rotation number $r\left(S_{a b}\right)$ is given by

$$
r\left(S_{a b}\right)=\frac{3 \pi-7 \theta}{14 \pi-32 \theta} .
$$

To establish this, we consider iteration $T_{J}^{(p)}$ of the first return map $T_{J}$ on the sector $J$. Pick $\mathbf{w} \in J$ lying on $E(r, \phi)$ and set

$$
T_{J}^{(p)}(\mathbf{w})=M_{1}^{m_{1}} M_{2}^{m_{2}} \mathbf{w}
$$

with $m_{1}=m_{1}(p), m_{2}=m_{2}(p)$, both going to $+\infty$ as $p \rightarrow \infty$. Since each appearance of $M_{1}$ or $M_{2}$ corresponds to one counterclockwise revolution of the circle, while $M_{1}$ and $M_{2}$ correspond to 5 and 4 iterations of $T_{a b}$, respectively, we have

$$
r\left(S_{a b}\right)=\lim _{p \rightarrow \infty} \frac{m_{1}(p)+m_{2}(p)}{5 m_{1}(p)+4 m_{2}(p)} .
$$

However the condition that the angle $\omega$ remains inside the sector $J$ of $E(r, \phi)$ at each iteration of $T_{J}$ yields

$$
0<\phi+m_{1}(p)(\pi-3 \theta)+m_{2}(p)(-2 \pi+4 \theta)<2 \pi .
$$


Letting $p \rightarrow \infty$, this constraint implies that

$$
\lim _{p \rightarrow \infty} \frac{m_{2}(p)}{m_{1}(p)}=\frac{\pi-3 \theta}{2 \pi-4 \theta} .
$$

Rewriting (6.7) as

$$
r\left(S_{a b}\right)=\lim _{p \rightarrow \infty} \frac{1+\frac{m_{2}(p)}{m_{1}(p)}}{5+4 \frac{m_{2}(p)}{m_{1}(p)}}
$$

and substituting (6.8) yields the desired rotation number formula (6.6).

Suppose that $r\left(S_{a b}\right)$ is rational. By (6.6) this occurs if and only if $\theta$ is a rational multiple of $\pi$. We show that $T_{a b}$ is periodic. Indeed, for rational rotation number $S_{a b}$ has a periodic orbit, and this orbit necessarily contains some point $\mathbf{w} \in J$. Since $S_{a b}^{(q)}(\mathbf{w})=\mathbf{w}$ where $q$ is the period, we have $T_{a b}^{(q)}(\mathbf{w})=\lambda \mathbf{w}$ for some real $\lambda$. Since this value is an iterate of a first return map to $J$, there is some $p$ such that

$$
T_{a b}^{(q)}(\mathbf{w})=T_{J}^{(p)}(\mathbf{w})=M_{1}^{m_{1}} M_{2}^{m_{2}} \mathbf{w} .
$$

Now $M_{1}^{m_{1}} M_{2}^{m_{2}}$ has $\mathbf{w}$ as an eigenvector with a real eigenvalue, but it also has $\mathbf{w}^{+}$and $\mathbf{w}^{-}$as eigenvectors with complex conjugate eigenvalues of norm 1 . It follows that $M_{1}^{m_{1}} M_{2}^{m_{2}}= \pm I$. so that

$$
T_{a b}^{(2 q)}(\mathbf{w})=T_{J}^{(2 r)}(\mathbf{w})=M_{1}^{2 m_{1}} M_{2}^{2 m_{2}} \mathbf{w} .
$$

It follows that the first return map $T_{J}^{(2 p)}$ is the identity on a subsector of $J$ of positive width, so $S_{a b}$ must have infinitely many periodic points of period $2 q$, and Theorem 2.1(iii) shows that $T_{a b}$ is periodic, with period dividing $2 q$.

Suppose that $r\left(S_{a b}\right)$ is irrational. Then Theorem 3.4 applies to show that $T_{a b}$ has a piecewise conic invariant circle with at most 8 pieces, and the pieces are arcs of ellipses by (6.5).

We consider the special parameters $a=-b=\sqrt[4]{2}$. Defining $\theta$ by $a=2 \cos \theta$ shows that $x=e^{i \theta}$ satisfies $x+1 / x=\sqrt[4]{2}$, hence it also satisfies

$$
x^{8}+4 x^{6}+4 x^{4}+4 x^{2}+1=0 .
$$

We show the rotation number $r\left(S_{a b}\right)$ is irrational by showing that $\frac{\theta}{\pi}$ is irrational, i.e. that $e^{i \theta}$ is not a root of unity. It suffices to show that (6.9) has no root that is a root of unity. One checks using MAPLE that this polynomial is irreducible over $\mathbb{Q}$, and that it is not a cyclotomic polynomial since it has a root off the unit circle. Thus it has no roots of unity as roots, and $r\left(S_{a b}\right)$ is irrational. Thus $T_{a b}$ has a piecewise elliptical invariant circle, pictured in Figure 4.1 .

Example 4.2, $T_{a b}$ with $0<a<1$, with $b=2 \frac{a^{2}-1}{a^{3}-2 a}$. Here $T_{a b}^{(10)}(0,1)=(0,-1)$.

Proof. We first verify that one has $T_{a b}^{(10)}(0,1)=(0,-1)$. Set $\mathbf{v}_{0}=(0,-1)$ and $\mathbf{v}_{j}=T_{a b}^{(j)}\left(\mathbf{v}_{0}\right)=$ $\mathbf{v}_{j}$; the signs $\left.S_{j}=\operatorname{Sign}\left(\mathbf{v}_{j}\right)_{x}\right)$ determine the sequence of iterates. The resulting $\mathbf{v}_{j}$ and their sign values are given in Table 6.2.

To verify the entries in Table 6.2, note that the condition $0<a<1$ and the given sign sequence determine $\left\{\mathbf{v}_{j}: 1 \leq j \leq 5\right\}$, and in particular

$$
\mathbf{v}_{6}=\left(a^{3} b^{2}+2 a^{2} b+2 a b^{2}-a-2 b, a^{2}+2 a b+a^{3} b-1\right)
$$


We impose the condition that $\mathbf{v}_{6}=R\left(\mathbf{v}_{5}\right)$, and this requires that

$$
\left(a^{3}-2 a\right) b^{2}-3\left(a^{2}-1\right) b+2 a=0 .
$$

The correct choice of root of this quadratic equation determines $b$ as given; one has $-\sqrt{2}<b<0$ and it produces the sign sequence as given above for $1 \leq j \leq 5$. As in Example 4.1 the relation $\mathbf{v}_{6}=R\left(\mathbf{v}_{5}\right)$ forces $\mathbf{v}_{j}=\mathbf{v}_{11-j}$ for $6 \leq j \leq 10$, and determines the remaining sign sequence, and gives $T_{a b}^{(10)}(0,-1)=(0,1)$.

We take $\mathcal{O}=\left\{\mathbf{v}_{j}: 1 \leq j \leq 10\right\}$, and the hypotheses of Theorem 3.3 and 3.4 apply whenever the rotation number $r\left(S_{a b}\right)$ is irrational. The elements of $\mathcal{O}$ appear in counterclockwise angular order as

$$
\mathbf{v}_{1}, \mathbf{v}_{10}, \mathbf{v}_{6}, \mathbf{v}_{2}, \mathbf{v}_{7}, \mathbf{v}_{3}, \mathbf{v}_{8}, \mathbf{v}_{9}, \mathbf{v}_{5} .
$$

We choose the sector $J=\mathbb{R}^{+}\left[\mathbf{v}, \mathbf{v}^{\prime}\right):=\mathbb{R}^{+}\left[\mathbf{v}_{1}, \mathbf{v}_{10}\right)$, which is exactly the third quadrant, with $\operatorname{sign}(-,-)$. The breakpoint is

$$
\mathbf{v}^{*}=\left(a^{2}-1,\left(a^{2}-1\right) b-a\right) .
$$

The first return maps on the subsectors $J_{1}=\mathbb{R}^{+}\left[\mathbf{v}, \mathbf{v}^{*}\right)$ and $J_{2}=\mathbb{R}^{+}\left[\mathbf{v}^{*}, \mathbf{v}^{\prime}\right)$ are well-defined regardless of whether the rotation number is rational or irrational.

The first return map on $J_{1}$ is $T_{a b}^{(4)}$ with symbol sequence $(-++-)$ and its associated matrix $M_{1}$ is:

$$
M_{1}=\left[\begin{array}{cc}
\left(a^{2}-1\right) b^{2}-2 a b+1 & \left(1-a^{2}\right) b+a \\
\left(a^{2}-1\right) b-a & 1-a^{2}
\end{array}\right] .
$$

The first return map on $J_{2}$ is $T_{a b}^{(13)}$ with symbol sequence $(-+++-+++-+++-)$ and its associated matrix $M_{2}$ is:

$$
M_{2}=\left[\begin{array}{cc}
\left(1-a^{2}\right) b+a & a^{2}-1 \\
1-a^{2} & \frac{a^{2}\left(a^{2}-2\right)}{\left(a^{2}-1\right) b-a}
\end{array}\right] .
$$

We have $\operatorname{Tr}\left(M_{1}\right)=\left(a^{2}-1\right)\left(b^{2}-1\right)-2 a b+1$ and using the relation (6.10) and the fact that $b<0$, we obtain ${ }^{2}$

$$
\operatorname{Tr}\left(M_{1}\right)=2 \text { for } a=\alpha_{0} \approx 0.3802,
$$

where $\alpha_{0}$ is the unique root in the unit interval of

$$
x^{4}+3 x^{3}+3 x^{2}+x-1=0 .
$$

One can check that $0<\operatorname{Tr}\left(M_{1}\right)<2$ for $0<a<\alpha_{0}$ and $\operatorname{Tr}\left(M_{1}\right)>2$ for $\alpha_{0}<a<1$.

Subcase 1. Piecewise hyperbolic case $\left(\alpha_{0}<a<1\right)$

The matrix $M_{1}$ is hyperbolic and has real eigenvectors and eigenvalues; therefore $M_{2}$ does also, since it commutes with $M_{1}$ and has the same eigenvectors. One checks $\operatorname{Tr}\left(M_{2}\right)>2$ as well. The first return map $T_{J}$ for $J$ leaves invariant hyperbolic arcs in $J$, even in the rational rotation

\footnotetext{
${ }^{2}$ Take the resultant of 6.10 and the polynomial $\operatorname{Tr}\left(M_{1}\right)-2$. It is a polynomial of degree 8 in $a$ which has two degree 4 factors over $\mathbb{Q}$. The other degree 4 factor produces extraneous roots.
} 
number case. These are bounded arcs, for one can verify numerically that the asymptotes of the hyperbola fall strictly outside $J$. Thus the orbit of a given point $\mathbf{w} \in J$, lies on some bounded hyperbolic $\operatorname{arc} \mathcal{C}_{1}$ inside $J$.

Let $\lambda_{1}=\lambda_{1}(a)>1$ be the large eigenvalue of $M_{1}$, the other eigenvalue being $1 / \lambda_{1}$; let $\lambda_{2}>1$ be the large eigenvalue of $M_{2}$, the other being $1 / \lambda_{2}$. We choose the eigenvectors so that $M_{1} \mathbf{w}_{+}=\lambda_{1} \mathbf{w}_{+}$and $M_{1} \mathbf{w}_{-}=1 / \lambda_{1} \mathbf{w}_{-}$. We now claim that

$$
M_{2} \mathbf{w}_{+}=\frac{1}{\lambda_{2}} \mathbf{w}_{+}
$$

To establish this, choose a point $\mathbf{w}$ in $J$, whose first return iterates $T_{J}^{(p)}$ necessarily all lie on a fixed hyperbolic $\operatorname{arc} \mathcal{C}_{1}$ in $J$. Write

$$
T_{J}^{(p)}(\mathbf{w})=M_{1}^{m_{1}} M_{2}^{m_{2}} \mathbf{w},
$$

with $m_{1}=m_{1}(p), m_{2}=m_{2}(p)$, both going to $+\infty$ as $p \rightarrow \infty$. Express $\mathbf{w}$ in terms of the eigenvectors as

$$
\mathbf{w}=c_{1} \mathbf{w}_{+}+c_{2} \mathbf{w}_{-},
$$

with $c_{1} c_{2} \neq 0$ since $\mathbf{w}$ cannot be an eigenvector. We now argue by contradiction, and suppose $M_{2} \mathbf{w}_{+}=\lambda_{2} \mathbf{w}_{+}$. Then

$$
T_{J}^{(p)}(\mathbf{w})=c_{1} \lambda_{1}^{m_{1}} \lambda_{2}^{m_{2}} \mathbf{w}_{+}+c_{2} \lambda_{1}^{-m_{1}} \lambda_{2}^{-m_{2}} \mathbf{w}_{-},
$$

The first term on the right gets large as $p \rightarrow \infty$, while the second gets small, hence $\left\|T_{J}^{(p)}(\mathbf{w})\right\| \rightarrow$ $\infty$ as $p \rightarrow \infty$. This contradicts $T_{J}^{(p)}(\mathbf{w}) \in \mathcal{C}_{1}$ for all $p$; the claim follows.

We show that the rotation number $r\left(S_{a b}\right)$ is given by

$$
r\left(S_{a b}\right)=\frac{\log \lambda_{2}+3 \log \lambda_{1}}{4 \log \lambda_{2}+13 \log \lambda_{1}} .
$$

We pick a point $\mathbf{w} \in J$ and study the first return map iterates, with $T_{J}^{(p)}(\mathbf{w})=M_{1}^{m_{1}} M_{2}^{m_{2}} \mathbf{w}$ and $m_{1}=m_{1}(p), m_{2}=m_{2}(p) \rightarrow \infty$ as $p \rightarrow \infty$. The return map for $J_{1}$ goes around the circle once, while that for $M_{2}$ goes around three times, and using the number of steps of $T_{a b}$ each takes we obtain

$$
r\left(S_{a b}\right)=\lim _{p \rightarrow \infty} \frac{m_{1}(p)+3 m_{2}(p)}{4 m_{1}(p)+13 m_{2}(p)} .
$$

Expressing $\mathbf{w}=c_{1} \mathbf{w}_{+}+c_{2} \mathbf{w}_{-}$in terms of eigenvectors and using (6.13) yields

$$
T_{J}^{(p)}(\mathbf{w})=c_{1} \lambda_{1}^{m_{1}} \lambda_{2}^{-m_{2}} \mathbf{w}_{+}+c_{2} \lambda_{1}^{-m_{1}} \lambda_{2}^{m_{2}} \mathbf{w}_{-} .
$$

In order for these vectors to remain on $\mathcal{C}_{1}$ their norms must be bounded away from 0 and $\infty$, so there is a positive constant $C$ such that $1 / C<\lambda_{1}^{m_{1}} \lambda_{2}^{-m_{2}}<C$. This yields

$$
\lim _{p \rightarrow \infty} \frac{m_{1}(p)}{m_{2}(p)}=\frac{\log \lambda_{2}}{\log \lambda_{1}}
$$

Substituting this in (6.15) yields the rotation number formula (6.14). 
When $r\left(S_{a b}\right)$ is rational, then $T_{a b}$ is periodic. To show this, we use the fact that the eigenvectors of $M_{1}$ and $M_{2}$ fall on rays lying outside the sector $J$. If $r\left(S_{a b}\right)$ is rational, then $S_{a b}$ has a periodic orbit, which necessarily visits the sector $J$. Choosing an element $\mathbf{w} \in J$ on this orbit, one has some first return value $T_{J}^{(p)}(\mathbf{w})=\lambda \mathbf{w}$, for some real value $\lambda$. Now $T_{J}^{(p)}(\mathbf{w})=M_{1}^{m_{1}} M_{2}^{m_{2}} \mathbf{w}$, and the matrix $M=M_{1}^{m_{1}} M_{2}^{m_{2}}$ now has three distinct eigenvectors, $\mathbf{w}_{+}, \mathbf{w}_{-}$and $\mathbf{w}$, so $M= \pm I$. Now we find that $T_{J}^{(2 p)}\left(\mathbf{w}^{\prime}\right)=M_{1}^{2 m_{1}} M_{2}^{2 m_{2}} \mathbf{w}^{\prime}=\mathbf{w}^{\prime}$ for all $\mathbf{w}^{\prime}$ on a subsector of positive angular width. It follows that $S_{a b}$ has infinitely many periodic orbits, so $T_{a b}$ is periodic by Theorem 2.1 (iii).

When $r\left(S_{a b}\right)$ is irrational, Theorem 3.4 applies to show that $T_{a b}$ has an invariant circle which is a piecewise union of at most 10 arcs of hyperbolas.

We consider the special parameter values $a=-b=\sqrt{\frac{\sqrt{5}-1}{2}}$. Here $\operatorname{Tr}\left(M_{1}\right)=a^{4}+2>2$. The larger eigenvalue $\lambda_{1}$ of $M_{1}$ satisfies $x^{2}-\left(a^{4}+2\right) x+1=0$, and hence one finds it is an algebraic unit that satisfies

$$
x^{4}-7 x^{3}+13 x^{2}-7 x+1=0 .
$$

A similar calculation shows that $\lambda_{2}$ is an algebraic unit that satisfies

$$
x^{8}+23 x^{6}-77 x^{4}+23 x^{2}+1=0 .
$$

The rotation number formula (6.14) gives

$$
r\left(S_{a b}\right)=\frac{\log \left(\lambda_{1}^{3} \lambda_{2}\right)}{\log \left(\lambda_{1}^{13} \lambda_{2}^{4}\right)}
$$

and the condition for $r\left(S_{a b}\right)$ to be irrational is that

$$
\lambda_{1}^{n_{1}} \lambda_{2}^{n_{2}} \neq 1 \text { for }\left(n_{1}, n_{2}\right) \in \mathbb{Z}^{2} \backslash(0,0) .
$$

That is, $\lambda_{1}$ and $\lambda_{2}$ must be multiplicatively independent algebraic units in the algbraic number field over $\mathbb{Q}$ that they generate. This can be verified by checking that their logarithms and the logarithms of the absolute values of their algebraic conjugates span a two dimensional lattice. Given that $r\left(S_{a b}\right)$ is irrational, Theorem 3.4 applies to show $T_{a b}$ has an invariant circle, which consists of at most 10 arcs of hyperbolas, as pictured in Figure 4.2

Given that $r\left(S_{a b}\right)$ is irrational, we can show it is transcendental. Clearing denominators in (6.16) and exponentiating gives

$$
\left(\lambda_{1}^{13} \lambda_{2}^{4}\right)^{r}=\lambda_{1}^{3} \lambda_{2}
$$

The right side of this equation is an algebraic number, and the left side is an algebraic number raised to the $r$-th power. If $r=r\left(S_{a b}\right)$ were an irrational algebraic number, then the left side would be transcendental by the criterion ${ }^{3}$ of Theorem 2.4 in Baker [2]. This is a contradiction, so we conclude that $r\left(S_{a b}\right)$ is transcendental.

Subcase 2. Piecewise linear case $\left(a=\alpha_{0}\right)$

\footnotetext{
${ }^{3}$ In [2] Theorem 2.4] take $\alpha_{1}=\lambda_{1}^{13} \lambda_{2}^{4}$ and $\beta=r$, and $[1, \beta]$ are linearly independent over $\mathbb{Q}$ since $r$ is irrational.
} 
Here $a=\alpha_{0}, b=\alpha_{0}^{3}+2 \alpha_{0}^{2}+\alpha_{0}-1$, where $\alpha_{0}$ satisfies (6.12). The sector $\left.J=\mathbb{R}^{+}[(-1,0)),(0,-1)\right)$ and in this case one finds that

$$
\begin{gathered}
M_{1}=I+\left[\begin{array}{cc}
\alpha_{0}^{2} & \alpha_{0}^{2} \\
-\alpha_{0}^{2} & -\alpha_{0}^{2}
\end{array}\right], \\
M_{2}=I+\left[\begin{array}{cc}
\alpha_{0}^{2}-1 & \alpha_{0}^{2}-1 \\
1-\alpha_{0}^{2} & 1-\alpha_{0}^{2}
\end{array}\right] .
\end{gathered}
$$

Consequently

$$
M_{1}^{m_{1}} M_{2}^{m_{2}}=I+\left[\begin{array}{cc}
x & x \\
-x & -x
\end{array}\right]
$$

with $x=\left(m_{1}+m_{2}\right) \alpha_{0}^{2}-m_{2}$. By Lemma 3.1 these commuting maps leave invariant a family of parallel straight lines, and in the sector $J$ the first return map iterates of any given point $\mathbf{w}$ lie on a certain finite line segment $\mathcal{C}_{1}$.

We show that the rotation number

$$
r\left(S_{a b}\right)=\frac{2 \alpha_{0}^{2}+1}{9 \alpha_{0}^{2}+4} .
$$

For this, picking a point $\mathbf{w} \in J$ and using the fact that the orbit $T_{J}^{(p)}(\mathbf{w})=M_{1}^{m_{1}} M_{2}^{m_{2}} \mathbf{w}$ Just as in subcase 1) we have

$$
r\left(S_{a b}\right)=\lim _{p \rightarrow \infty} \frac{m_{1}(p)+3 m_{2}(p)}{4 m_{1}(p)+13 m_{2}(p)} .
$$

Next, using the fact that the orbit $T_{J}^{(p)}(\mathbf{w})$ lies on the line segment $\mathcal{C}_{1}$, so has norm bounded away from 0 and $\infty$, using (6.17) we have $\left|m_{1}(p) \alpha_{0}^{2}+m_{2}(p)\left(\alpha_{0}^{2}-1\right)\right|<C$ for some positive $C$. Letting $p \rightarrow \infty$ yields

$$
\lim _{p \rightarrow \infty} \frac{m_{1}(p)}{m_{2}(p)}=\frac{1-\alpha_{0}^{2}}{\alpha_{0}^{2}}
$$

and substituting this in (6.19) yields (6.18).

The rotation number $r\left(S_{a b}\right)$ is irrational, as is verified by computing the irreducible polynomial it satisfies over $\mathbb{Q}$, which has degree 4. Now Theorem 3.4 applies to show that $T_{a b}$ has a piecewise linear invariant circle with at most 10 pieces. This justifies Figure 4.3

Subcase 3. Piecewise ellipse case $\left(0<a<\alpha_{0}\right)$

This case is analyzed similarly to the case of Example 4.1 and we omit the details. The invariant circle is piecewise conic with arcs of ellipses when the rotation number is irrational, and $T_{a b}$ is periodic when the rotation number is rational.

Consider the special parameter values $a=\frac{1}{10}$, where $b=5\left(\frac{297-\sqrt{89801}}{199}\right)$. One finds that the (complex conjugate) eigenvalues $\lambda_{1}, \bar{\lambda}_{1}$ of $M_{1}$ and $\lambda_{2}, \bar{\lambda}_{2}$ of $M_{2}$ are roots of degree 4 polynomials over $\mathbb{Z}[x]$ with large coefficients. Verifying irrationality of the rotation number $r\left(S_{a b}\right)$ reduces to showing that $\left\langle\lambda_{1}, \lambda_{2}\right\rangle$ generate multiplicatively a free abelian group of rank 2 . These numbers are not algebraic integers, and it suffices to give a set of prime ideals such that the 
valuations of $\lambda_{1}$ and $\lambda_{2}$ at those primes span a lattice of rank 2. In particular, at the four prime ideals above $2, \lambda_{1}$ has valuations $(2,-2,2,-2)$, while $\lambda_{2}$ has valuations $(7,-3,3,-7)$. Thus $\lambda_{1}^{i} \lambda_{2}^{j}$ is an algebraic integer only when $i=j=0$. The irrationality of $r\left(S_{a b}\right)$ shows that this $T_{a b}$ has a piecewise elliptic invariant circle, as pictured in Figure 4.4

Example 4.3. $T_{a b}$ with $1<a<\sqrt{2}$, with $b=\frac{(a-1)\left(2 a^{3}-4 a-1\right)}{a\left(a^{2}-2\right)\left(a^{2}-a-1\right)}$. Here $T_{a b}^{(13)}(0,-1)=(0,1)$.

Proof. One verifies that one has $T_{a b}^{(13)}(0,-1)=(0,1)$ in a manner similar to the earlier examples. Set $\mathbf{v}_{0}=(0,-1)$ and $\mathbf{v}_{j}=T_{a b}^{(j)}\left(\mathbf{v}_{0}\right)$, and define the sequence of symbols $S_{j}=$ $\operatorname{Sign}\left(\mathbf{v}_{j}\right)_{x}$ which determine the sequence of iterates. The resulting $\mathbf{v}_{j}$ and their symbol values $S_{j}$ are given in Table 6.3 The value $b=\frac{(a-1)\left(2 a^{3}-4 a-1\right)}{a\left(a^{2}-2\right)\left(a^{2}-a-1\right)}$ is determined by the requirement that $R\left(\mathbf{v}_{7}\right)=\mathbf{v}_{7}$, plus the given sign seqence $\left\{S_{i}: 1 \leq i \leq 6\right\}$. This value of $b$ has been substituted in the earlier iterates; one verifies retrospectively that the symbol sequence $\left.S_{j}: 1 \leq \mathbf{v}_{j} \leq 6\right\}$ is as indicated.

The points appear around the circle in counterclockwise order

$$
\mathbf{v}_{1}, \mathbf{v}_{6}, \mathbf{v}_{11}, \mathbf{v}_{2}, \mathbf{v}_{7}, \mathbf{v}_{12}, \mathbf{v}_{3}, \mathbf{v}_{8}, \mathbf{v}_{13}, \mathbf{v}_{4}, \mathbf{v}_{9}, \mathbf{v}_{5}, \mathbf{v}_{10}
$$

Of the thirteen arcs these determine, five have a well-defined first return map and eight do not. The ones that do are divided into two intervals by a breakpoint and the conclusion of Theorem 3.3 holds for them; they are the intervals with left endpoint $\mathbf{v}_{9}, \mathbf{v}_{10}, \mathbf{v}_{11}, \mathbf{v}_{12}, \mathbf{v}_{13}$, respectively. The other eight intervals are $\left[v_{i}, v_{i+5}\right)$ for $1 \leq i \leq 8$; they are the images under $T_{a b}^{(i)}$ of the interval $\left[(0,-1), v_{5}\right), 1 \leq i \leq 8$. These intervals are entirely transient; that is, any orbit contains at most one point from each of the eight intervals.

We consider the interval $J=\mathbb{R}^{+}\left[\mathbf{v}, \mathbf{v}^{\prime}\right):=\mathbb{R}^{+}\left[\mathbf{v}_{9}, \mathbf{v}_{5}\right)$. The break point $\mathbf{v}^{*}=(0,-1)$. The first return map on $J_{1}=\left[\mathbf{v}, \mathbf{v}^{*}\right)$ is $T_{a b}^{(5)}$ and has symbol sequence $(-+++-)$; its associated matrix $M_{1}$ is

$$
M_{1}=\left[\begin{array}{cc}
\left(a^{3}-2 a\right) b^{2}+\left(-2 a^{2}+2\right) b+a & \left(-a^{3}+2 a\right) b+a^{2}-1 \\
\left(a^{3}-2 a\right) b-a^{2}+1 & -a^{3}+2 a
\end{array}\right] .
$$

The first return map on $J_{2}=\left[\mathbf{v}^{*}, \mathbf{v}^{\prime}\right)$ is $T_{a b}^{(9)}$ and has symbol sequence $(++++-++++)$; its associated matrix $M_{2}$ is

$$
M_{2}=\frac{a^{2}-a-1}{a-1} M_{1}+\frac{a^{2}(a-2)\left(a^{2}-2\right)^{2}}{(a-1)\left(a^{2}-a-1\right)} I .
$$

The argument of Theorem 3.3 applies to show these two matrices commute. One checks that $\left|\operatorname{Tr}\left(M_{1}\right)\right|>2$ for $1<a<\sqrt{2}$, hence the associated matrices are hyperbolic.

We show that the rotation number

$$
r\left(T_{a b}\right)=\frac{1}{5}
$$

This follows because the subsector $J^{\prime}=\mathbb{R}^{+}[(-1,0),(-1,-1))$ is mapped into itself by $T_{J}$ and therefore contains an attracting periodic point; since it is in $J_{1}$ this point has periodic symbol sequence $(-+++-)$ and therefore has rotation number $1 / 5$. 


\begin{tabular}{|l|r|}
\hline \multicolumn{1}{|c|}{ vector } & $\operatorname{Sign}\left(\mathbf{v}_{j}\right)_{x}$ \\
\hline $\mathbf{v}_{1}=(-1,0)$ & - \\
$\mathbf{v}_{2}=(-b,-1)$ & + \\
$\mathbf{v}_{3}=(-a b+1,-b)$ & + \\
$\mathbf{v}_{4}=\left(-a^{2} b+a+b, 1-a b\right)$ & + \\
$\mathbf{v}_{5}=\left(a^{3} b+a^{2}+2 a b-1,-a^{2} b+a+b\right)$ & - \\
$\mathbf{v}_{6}=\left(-a^{2} b+a+b, a^{3} b+a^{2}+2 a b-1\right)$ & + \\
$\mathbf{v}_{7}=\left(-a b+1,-a^{2} b+a+b\right)$ & + \\
$\mathbf{v}_{8}=(-b,-a b+1)$ & + \\
$\mathbf{v}_{9}=(-1,-b)$ & - \\
$\mathbf{v}_{10}=(0,-1)$ & \pm \\
\hline
\end{tabular}

Table 6.2: Orbit $\mathcal{O}$ for example 4.2

\begin{tabular}{|l|r|}
\hline \multicolumn{1}{|c|}{ vector } & $\operatorname{Sign}\left(\mathbf{v}_{j}\right)_{x}$ \\
\hline $\mathbf{v}_{1}=(1,0)$ & + \\
$\mathbf{v}_{2}=(a, 1)$ & + \\
$\mathbf{v}_{3}=\left(a^{2}-1, a\right)$ & + \\
$\mathbf{v}_{4}=\left(a\left(a^{2}-2\right), a^{2}-1\right)$ & - \\
$\mathbf{v}_{5}=\left(a(a-1)\left(a^{2}-2\right) /\left(a^{2}-a-1\right), a\left(a^{2}-2\right)\right.$ & + \\
$\mathbf{v}_{6}=\left(a\left(a^{2}-2\right) /\left(a^{2}-a-1\right), a(a-1)\left(a^{2}-2\right) /\left(a^{2}-a-1\right)\right)$ & + \\
$\mathbf{v}_{7}=\left(a\left(a^{2}-2\right) /\left(a^{2}-a-1\right), a\left(a^{2}-2\right) /\left(a^{2}-a-1\right)\right)$ & + \\
$\mathbf{v}_{8}=\left(a(a-1)\left(a^{2}-2\right) /\left(a^{2}-a-1\right), a\left(a^{2}-2\right) /\left(a^{2}-a-1\right)\right)$ & + \\
$\mathbf{v}_{9}=\left(a\left(a^{2}-2\right), a(a-1)\left(a^{2}-2\right) /\left(a^{2}-a-1\right)\right)$ & - \\
$\left.\mathbf{v}_{10}=\left(a^{2}-1\right), a\left(a^{2}-2\right)\right)$ & + \\
$\mathbf{v}_{11}=\left(a, a^{2}-1\right)$ & + \\
$\left.\mathbf{v}_{12}=(1, a)\right)$ & + \\
$\mathbf{v}_{13}=(0,1)$ & \pm \\
\hline
\end{tabular}

Table 6.3: Orbit $\mathcal{O}$ for example 4.3 
Lemma 3.1 applies to show that on the sector $J$ the iterates of a given point lie on arcs of a hyperbola, or on asymptotes. One can show that the interval $J_{1}$ contains two asymptotes of a hyperbola. Each of these defines a periodic point of order 5, one attracting and one repelling, defining the two periodic orbits of period 5 of $S_{a b}$. It follows that the first return map orbits of all points diverge in norm to $+\infty$ under forward iterations, except for those on the repelling fixed ray, which go to $+\infty$ under backwards iteration.

The points in $J$ not on an asymptote under iteration of the first return map will approach an asymptote, which is an attracting fixed point of the first return map. For the map $T_{a b}$ it is an attracting periodic orbit, and there are five copies of the asymptotes. The orbit of any point not on a periodic orbit of $S_{a b}$ is contained in a finite union of arcs of hyperbolas, even though there are no invariant circles. Theorem 3.4 does not apply to bound the number of pieces, because the first return map on $J$ has three pieces; however the number of pieces appears to be at most 23 .

\section{References}

[1] V. I. Arnold and A. Avez, Ergodic Problems of Classical Mechanics, W. A. Benjamin: New York 1968.

[2] A. Baker, Transcendental Number Theory, Cambridge Univ. Press, Cambridge 1975.

[3] A. F. Beardon, S. R. Bullett and P. J. Rippon, Periodic orbits of difference equations, Proc. Roy. Soc. Edinburgh 125A 91995), 657-674.

[4] C. Froeschlè, Étude numérique de transformations ponctuelles planes conservant les aires, C. R. Acad. Sci. Paris 266 (1968), 846-848.

[5] M. Herman, Sur la conjugasion differentiable des difféomorphismes du cercle. Publ. Math. IHES 49 (1979), 5-234.

[6] M. Herman, Sur les Courbes Invariantes par les Difféomorphismes de l'Anneau. Vol. 2, Astérisque 144, Soc. Math. de France: Paris 1986.

[7] J. C. Lagarias and E. Rains, Dynamics of a family of piecewise-linear area-preserving plane maps I. Rational rotation numbers, eprint: arxiv:math.DS/0301294

[8] J. C. Lagarias and E. Rains, Dynamics of a family of piecewise-linear area-preserving plane maps III. Cantor set spectra, eprint: arxiv:math.DS/0505103

[9] V. F. Lazutkin, KAM Theory and Semiclassical Approximations to Eigenfunctions, Springer-Verlag: Berlin 1993. 